\title{
Lepton flavor violation without supersymmetry
}

\author{
V. Cirigliano, A. Kurylov, M. J. Ramsey-Musolf, and P. Vogel \\ Kellogg Radiation Laboratory, California Institute of Technology, Pasadena, California 91125, USA
}

(Received 29 April 2004; published 11 October 2004)

\begin{abstract}
We study the lepton flavor-violating (LFV) processes $\mu \rightarrow e \gamma, \mu \rightarrow 3 e$, and $\mu \rightarrow e$ conversion in nuclei in the left-right symmetric model without supersymmetry and perform the first complete computation of the LFV branching ratios $B(\mu \rightarrow f)$ to leading nontrivial order in the ratio of leftand right-handed symmetry-breaking scales. To this order, $B(\mu \rightarrow e \gamma)$ and $B(\mu \rightarrow e)$ are governed by the same combination of LFV violating couplings, and their ratio is naturally of order unity. We also find $B(\mu \rightarrow 3 e) / B(\mu \rightarrow e) \sim 100$ under slightly stronger assumptions. Existing limits on the branching ratios already substantially constrain mass splittings and/or mixings in the heavy neutrino sector. When combined with future collider studies and precision electroweak measurements, improved limits on LFV processes will test the viability of low-scale, nonsupersymmetric LFV scenarios.
\end{abstract}

DOI: 10.1103/PhysRevD.70.075007

PACS numbers: 12.10.Dm

\section{INTRODUCTION}

Leptons of different flavors do not mix in the Standard Model (SM) of electroweak interactions as a consequence of vanishing neutrino masses. The observation of neutrino oscillations, however, has provided clear evidence that nature does not conserve lepton flavor and that the SM must be part of a more fundamental theory that allows for lepton flavor violation (LFV). In the most widely held theories of neutrino mass, LFV is generated at high scales that are well beyond the reach of present and future collider experiments. Searching for LFV among charged leptons in low-energy measurements is an alternative and important way to probe additional aspects of LFV at such high scales.

Attempts to observe, and theoretically predict, the manifestations of LFV involving various modes of muon decay have a long tradition. The rather small upper limit $\left(2 \times 10^{-5}\right)$ on the branching ratio for the $\mu \rightarrow e+$ $\gamma$ decay determined by Lokanathan and Steinberger [1] almost 50 years ago led to a flurry of theoretical activity (see, e.g, [2] and many subsequent papers) that resulted in the realization that the electron and muon neutrinos are different particles-a fact confirmed experimentally shortly afterward. Over the intervening years the increase in the intensity of muon beams and advances of experimental techniques led to impressive improvement in the sensitivity of various searches for LFV. Even though no positive effects have been seen so far, the upper limits of the corresponding branching ratios became smaller by a factor of $\sim 10^{6}$. At present, the most stringent limit on the branching ratio for $\mu \rightarrow e \gamma$ is [3]

$$
B_{\mu \rightarrow e \gamma} \equiv \frac{\Gamma\left(\mu^{+} \rightarrow e^{+} \gamma\right)}{\Gamma\left(\mu^{+} \rightarrow e^{+} \nu \bar{\nu}\right)}<1.2 \times 10^{-11} \quad 90 \% \text { C.L. }
$$

obtained by the MEGA collaboration, while for the process of $\mu \rightarrow e$ conversion in gold nuclei, the SINDRUM collaboration has obtained the limit [4]

$$
\begin{aligned}
& B_{\mu \rightarrow e}^{A} \equiv \frac{\Gamma\left[\mu^{-}+A(N, Z) \rightarrow e^{-}+A(N, Z)\right]}{\Gamma\left[\mu^{-}+A(Z, N) \rightarrow \nu_{\mu}+A(Z-1, N+1)\right]} \\
& <8 \times 10^{-13} \quad 90 \% \text { C.L.. }
\end{aligned}
$$

The present limits on other branching ratios are similarly impressive: $1.0 \times 10^{-12}$ for $B_{\mu^{+} \rightarrow e^{+} e^{-} e^{+}}[5], 4.3 \times 10^{-12}$ for $B_{\mu \rightarrow e}^{\mathrm{Ti}}[6]$, and $4.6 \times 10^{-11}$ for $B_{\mu \rightarrow e}^{\mathrm{Pb}}$ [7]. Two ambitious new experiments aiming at substantial improvement in the sensitivity are being developed: MEG plans to reach sensitivity of $\sim 5 \times 10^{-14}$ for $B_{\mu^{+} \rightarrow e^{+}+\gamma}$ [8], while MECO aims to reach $\sim 5 \times 10^{-17}$ for $B_{\mu \rightarrow e}^{\mathrm{Al}}$ in aluminum [9].

Theoretically, the focus in recent years has been on frameworks that could both account for neutrino mass generation at high scales and lead to observable LFV in future experiments with charged leptons. The direct effects of light neutrinos on charged lepton LFV are "“GIM suppressed" by factors of $\left(\Delta m_{\nu}^{2} / M_{W}^{2}\right)^{2} \lesssim 10^{-50}$ in the rate and are, thus, entirely negligible. In order to obtain LFV effects that could be seen by experiment, a mechanism must exist for overcoming this GIM suppression. Such a mechanism necessarily involves physics at mass scales heavier than the weak scale. The primary motivation for LFV studies involving charged leptons is to help determine both the relevant scale as well as the most viable models associated with it.

Although a variety of such models have been considered, based on various supersymmetry (SUSY) scenarios [10-16], or left-right symmetry [17,18], the most commonly-quoted are SUSY grand unified theories (GUTs), wherein quarks and leptons are assigned to the same representation of the unification gauge group at the GUT scale. Consequently, the large Yukawa coupling responsible for the top quark mass also appears in LFV couplings $[13,14]$. The latter then give rise-via renormalization group evolution-to sizable lepton flavor nondiagonal soft SUSY-breaking terms at the $\mathrm{TeV}$ scale. Superpartner loops that contain insertion of these terms 
then produce unsuppressed LFV transitions involving charged leptons. For example, in a SUSY SU(5) scenario, one has [13]

$$
\begin{gathered}
B_{\mu \rightarrow e \gamma}=2.4 \times 10^{-12}\left(\frac{\left|V_{t s}\right|}{0.04} \frac{\left|V_{t d}\right|}{0.01}\right)^{2}\left(\frac{100 \mathrm{GeV}}{m_{\tilde{\mu}}}\right)^{4} \\
B_{\mu \rightarrow e}^{\mathrm{Ti}}=5.8 \times 10^{-12} \alpha\left(\frac{\left|V_{t s}\right|}{0.04} \frac{\left|V_{t d}\right|}{0.01}\right)^{2}\left(\frac{100 \mathrm{GeV}}{m_{\tilde{\mu}}}\right)^{4},
\end{gathered}
$$

neglecting gaugino masses. For superpartner masses of order the weak scale, one would expect to see nonzero signals in the up-coming MECO and MEG experiments under this scenario. Moreover, one would also expect to observe an order $\alpha$ suppression of $B_{\mu \rightarrow e}^{A}$ relative to $B_{\mu \rightarrow e \gamma}$ in this case since the conversion process entails the exchange of a virtual gauge boson between leptons and the nucleus rather than emission of a real photon.

In this paper, we study an alternative paradigm for LFV, wherein LFV occurs at much lower scales and does not require the presence of supersymmetric interactions to overcome the GIM suppression factor. In this scenario, neutrino mass generation occurs at the multi$\mathrm{TeV}$ scale via a spontaneously broken extended gauge group ${ }^{1}$. LFV for charged leptons arises from the interactions of the additional gauge bosons, heavy neutrinos, and Higgs bosons associated with the extended gauge symmetry. As an explicit realization of this scenario, we work within the left-right symmetric model (LRSM) [21-23], which gives a minimal, nonsupersymmetric extension of the SM with nonsterile, right-handed Majorana neutrinos. As such, it contains triplet Higgs fields that have nonzero hypercharge and that provide the simplest mechanism for generating a Majorana mass term [24]. As pointed out in Ref. [25], models of this type may give rise to unsuppressed operators for the LFV decay $\mu \rightarrow 3 e$, and these operators in turn induce logarithmicallyenhanced amplitudes for $\mu \rightarrow e$ conversion at loop level ${ }^{2}$. In the present case such effects, which were missed in earlier LRSM studies [17,18], result from the presence of the triplet Higgs fields. The large logarithms can compensate for the $\mathcal{O}(\alpha)$ suppression of $B_{\mu \rightarrow e}^{A}$ relative to $B_{\mu \rightarrow e \gamma}$ that generically follows for SUSY GUTs, and for Higgs masses of order $10 \mathrm{TeV}$ or below, both branching ratios may be large enough to be seen in future measurements. Roughly speaking, we find

$$
B_{\mu \rightarrow e \gamma} \approx 10^{-7} \times\left|g_{\text {lfv }}\right|^{2}\left(\frac{1 \mathrm{TeV}}{M_{W_{R}}}\right)^{4}
$$

\footnotetext{
${ }^{1}$ For a discussion of neutrino masses in this scenario and related phenomenological issues, see, e.g., Refs. [19,20].

${ }^{2}$ Specific realizations of these ideas been discussed for a doubly-charged scalar singlet [25] and R-parity-violating SUSY [15].
}

$$
B_{\mu \rightarrow e}^{\mathrm{Al}} \approx 10^{-7} \times \alpha\left|g_{\text {lfv }}\right|^{2}\left(\frac{1 \mathrm{TeV}}{M_{\delta_{R}^{++}}}\right)^{4}\left(\log \frac{M_{\delta_{R}^{++}}^{2}}{m_{\mu}^{2}}\right)^{2},
$$

where $M_{W_{R}}$ is the mass of the right-handed charged gauge boson, $M_{\delta_{R}^{++}}$is the mass of a doubly-charged, $S U(2)_{R}$ triplet Higgs, and

$$
g_{\text {lfv }}=\sum_{N}\left(K_{R}^{\dagger}\right)_{e N}\left(K_{R}\right)_{N \mu}\left(M_{N} / M_{W_{R}}\right)^{2},
$$

with $K_{R}$ being a flavor mixing matrix for the righthanded neutrinos of masses $M_{N}$ (see below). In the limit of degenerate, right-handed neutrinos, the LFV factor $g_{\text {lfv }}=0$. For heavy masses at the TeV scale, present experimental limits already constrain this factor to be tiny: $\left|g_{\text {lfv }}\right| \lesssim 10^{-2}$. In this case, the heavy neutrino spectrum must either be nearly degenerate or devoid of significant flavor mixing.

Note that both branching ratios are proportional to the same LFV factor, $\left|g_{\text {lfv }}\right|^{2}$. Naively, one would expect the loop graphs giving rise to $\mu \rightarrow e \gamma$ (with heavy neutrinogauge boson intermediate states) and the logarithmicallyenhanced loops that dominate $\mu \rightarrow e$ to have different prefactors. As discussed in more detail below, however, the doubly-charged, triplet Higgs $\delta_{R}^{++}$and its left-handed companion can have LFV violating Yukawa couplings $h_{i j}$ of $\mathcal{O}(1)$, but the sum over intermediate states in the logarithmically-enhanced loop graphs converts the sum over products of these couplings into $g_{\text {lfv }}$.

Should $g_{\text {lfv }}$ turn out to be nonzero, then one would expect the two branching ratios to be of similar size since the product of the $\ln ^{2}$ and $\alpha$ in $B_{\mu \rightarrow e}$ is $\mathcal{O}(1)$. We expect that any theory with nonsterile heavy Majorana neutrinos will contain such log enhancements, due to the presence of a more complicated Higgs sector than one finds in the SM. However, in SUSY GUT scenarios where LFVoccurs at high scales, these logarithmically-enhanced loop effects decouple below the GUT scale and do not affect the relative magnitudes of the branching ratios. Only when the symmetry-breaking scale is relatively light does one expect the two branching ratios to be commensurate in magnitude.

Somewhat weaker statements about the relationship between $B_{\mu \rightarrow e}$ and $B_{\mu \rightarrow 3 e}$ can also be made within the context of this model. In particular, we find

$$
B_{\mu \rightarrow 3 e} \approx 300 \times \frac{\left|h_{\mu e} h_{e e}^{*}\right|^{2}}{\left|g_{\text {lfv }}\right|^{2}} \times B_{\mu \rightarrow e}^{\mathrm{Al}},
$$

so that if all of the triplet Higgs couplings $h_{i j}$ are of roughly the same size and no cancellations occur in the sum $g_{\text {lfv }}=\sum_{j} h_{\mu j} h_{j e}^{*}$, the $\mu \rightarrow 3 e$ branching ratio should be roughly 2 orders of magnitude larger than the conversion ratio. Given the present experimental limits on $B_{\mu \rightarrow 3 e}$, one would then expect $B_{\mu \rightarrow e}^{\mathrm{Al}}$ to be of order $10^{-14}$ or smaller. As we discuss below, if the conversion 
ratio is found to be nonzero with significantly larger magnitude, then one would also expect to see a sizable effect in the channel $\tau \rightarrow 3 \ell$ (where $\ell$ denotes a charged lepton).

Finally, we observe that, while the logarithmic enhancement of $B_{\mu \rightarrow e}^{A}$ is a generic feature of any model that yields effective $\mu \rightarrow 3 e$ operators at tree-level, precise relationships between the various LFV observables depend on details of the model. In this respect, our perspective differs somewhat from the view in Ref. [25]. Indeed, the presence of a common factor of $\left|g_{\text {lfv }}\right|^{2}$ in $B_{\mu \rightarrow e \gamma}$ and $B_{\mu \rightarrow e}^{A}$-but not $B_{\mu \rightarrow 3 e}$-and its relation to the heavy neutrino spectrum follows from the pattern of symmetry-breaking in this scenario and the corresponding hierarchy of scales that enters the couplings of the right-handed gauge sector to matter. In order to implement this hierarchy in a self-consistent way, we adopt a power counting in $\kappa / v_{R}$, where $v_{R}$ and $\kappa$ are the scales, respectively, at which $S U(2)_{R}$ and electroweak symmetry are broken. In contrast to previous studies [17,18], we compute all LFV contributions through leading, nontrivial order in $\kappa / v_{R}$ and show that they decouple in the $v_{R} \rightarrow \infty$ limit as one would expect on general grounds [26]. In addition, we point out the prospective implications of other precision measurements and future collider studies for LFV in this scenario and vice-versa. The identification of such implications necessarily requires the adoption of a specific model, as the corresponding symmetries of the model dictate relationships between the coefficients of effective operators that would appear in an effective field theory framework. Thus, it is useful to have in hand a comprehensive treatment within various model frameworks in order to use experiment to discriminate among them. In R-parity-violating SUSY, for example, the LFV couplings that generate $\mu \rightarrow e$, etc., also appear, in general, in the mass matrices for light neutrino flavors [27], whereas in the LRSM LFV for charged leptons and light neutrinos are effectively independent.

Our discussion of the calculation is organized in the remainder of the paper as follows. In Section II we review the main features of the LRSM and define the relevant quantities. In Section III the effective vertices are calculated and the effective Lagrangians for the LFV processes are determined. Some of the detailed formulas are collected in the Appendices. Section IV gives an analysis of the results, along with a discussion of the rates as well as their ratios. We conclude in Section V.

\section{THE MODEL}

The gauge group of the theory is $\mathrm{SU}(2)_{\mathrm{L}} \times \mathrm{SU}(2)_{\mathrm{R}} \times \mathrm{U}(1)_{\mathrm{B}-\mathrm{L}}$ with the gauge couplings $g_{L}=g_{R}=g$ for the two SU(2)s and $g^{\prime}$ for the U(1). In this paper we follow the notation developed in Ref. [28] where the LRSM, its quantization, and its Feynman rules are discussed in detail. Below, we give a very brief introduction to the model, and explicitly define the quantities used in subsequent analysis.

The matter fields of the model include leptons $\left(L_{L, R}\right)$ and quarks $\left(Q_{L, R}\right)$, which are placed in the following multiplets of the gauge group:

$$
\begin{aligned}
L_{i L} & =\left(\begin{array}{c}
\nu_{i}^{\prime} \\
l_{i}^{\prime}
\end{array}\right)_{L}:(1 / 2: 0:-1), \\
L_{i R} & =\left(\begin{array}{c}
\nu_{i}^{\prime} \\
l_{i}^{\prime}
\end{array}\right)_{R}:(0: 1 / 2:-1), \\
Q_{i L} & =\left(\begin{array}{c}
u_{i}^{\prime} \\
d_{i}^{\prime}
\end{array}\right)_{R}:(1 / 2: 0: 1 / 3), \\
Q_{i R} & =\left(\begin{array}{c}
u_{i}^{\prime} \\
d_{i}^{\prime}
\end{array}\right)_{R}:(0: 1 / 2: 1 / 3) .
\end{aligned}
$$

Here, $i=1,2,3$ stands for generation number, and $\left(I_{L}, I_{R}, Y \equiv B-L\right)$ labels representation of the gauge group for each multiplet. The representation determines interactions of the multiplet with gauge fields. Before spontaneous symmetry-breaking (SSB) the latter include $W_{L}^{a, \mu}, W_{R}^{a, \mu}(a=1,2,3)$, and $B^{\mu}$ for $\mathrm{SU}(2)_{\mathrm{L}}, \mathrm{SU}(2)_{\mathrm{R}}$, and $\mathrm{U}(1)_{\mathrm{B}-\mathrm{L}}$ gauge group factors, respectively.

The SSB is achieved via the Higgs mechanism. The Higgs sector of the theory is not unique. However, the main results of this paper are largely independent of the details of the Higgs sector provided the LRSM has triplet Higgses and therefore heavy right-handed neutrinos. In our study we choose [22,23] a Higgs sector that consists of the bi-doublet $\phi:(1 / 2,1 / 2,0)$ and two triplets $\Delta_{L}:(1,0,2)$ and $\Delta_{R}:(0,1,2)$ :

$$
\begin{gathered}
\phi=\left(\begin{array}{cc}
\phi_{1}^{0} & \phi_{2}^{+} \\
\phi_{1}^{-} & \phi_{2}^{0}
\end{array}\right), \\
\Delta_{L, R}=\left(\begin{array}{cc}
\delta_{L, R}^{+} / \sqrt{2} & \delta_{L, R}^{++} \\
\delta_{L, R}^{0} & -\delta_{L, R}^{+} / \sqrt{2}
\end{array}\right), \\
\langle\phi\rangle=\left(\begin{array}{cc}
\kappa_{1} / \sqrt{2} & 0 \\
0 & \kappa_{2} / \sqrt{2}
\end{array}\right), \quad\left\langle\Delta_{L, R}\right\rangle=\left(\begin{array}{cc}
0 & 0 \\
v_{L, R} & 0
\end{array}\right),
\end{gathered}
$$

where the vacuum expectation values (VEVs) are shown in the second line. The most general Higgs potential with this field content has been analyzed in Ref. [19]. If one requires the scale $v_{R}$ in the multi-TeV range (but not significantly larger), the only choice which avoids excessive fine-tuning and leads to acceptable phenomenology is to set to zero certain couplings in the Higgs potential as well as $v_{L}$ [19]. Moreover, we assume no explicit or spontaneous $C P$ violation in the Higgs sector [29]. In summary, two distinct mass scales appear in the model: the electroweak symmetry-breaking scale $\kappa \sim \kappa_{1} \sim$ 
$\kappa_{2} \sim 250 \mathrm{GeV}$, and the scale $v_{R}$ at which $S U(2)_{R}$ and $U(1)_{B-L}$ are spontaneously broken. Phenomenological considerations require $v_{R} \gg \kappa$.

\section{A. Physical Fields}

After SSB matter and gauge fields acquire nonvanishing masses, which generally allow for mixing of the fields with the same quantum numbers. In the following, we identify masses and mixing angles which are important for our calculation. We omit the discussion of the quark sector of the model, as it is irrelevant for our work.

\section{Leptons}

The $3 \times 3$ mass matrix for charged leptons is $M_{l}=$ $\left(y_{D} \kappa_{2}+\tilde{y}_{D} \kappa_{1}\right) / \sqrt{2}$, where $y_{D}$ and $\tilde{y}_{D}$ are, respectively, the Yukawa coupling matrices for the bi-doublet $\phi$ and its charge conjugate. $M_{l}$ is diagonalized by a biunitary transformation $V_{L}^{l \dagger} M_{l} V_{R}^{l}=\left(M_{l}\right)_{\text {diag }}$. Since $\left(M_{l}\right)_{\text {diag }} \ll \kappa$, one has $y_{D}, \tilde{y}_{D} \ll 1$. Here, $V_{L, R}^{l}$ are $3 \times 3$ unitary matrices. These matrices relate the charged lepton mass eigenstates $l_{L, R}$ to the corresponding flavor eigenstates from Eq. (9): $l_{L, R}^{\prime}=V_{L, R}^{l} l_{L, R}$.

Within the LRSM it is convenient to describe neutrino fields by four-component spinors

$$
n_{R}^{\prime}=\left(\begin{array}{c}
\nu_{R}^{\prime c} \\
\nu_{R}^{\prime}
\end{array}\right), \quad n_{L}^{\prime}=\left(\begin{array}{c}
\nu_{L}^{\prime} \\
\nu_{L}^{\prime c}
\end{array}\right), \quad \nu_{L, R}^{\prime c}=i \sigma_{2} \nu_{R, L}^{\prime *} .
$$

The $6 \times 6$ mass matrix for the neutrinos is of see-saw type. It has both Majorana and Dirac entries:

$$
\begin{gathered}
M_{\nu}=\left(\begin{array}{cc}
0 & M_{D} \\
M_{D}^{T} & M_{R}
\end{array}\right), \quad M_{D}=\frac{1}{\sqrt{2}}\left(y_{D} \kappa_{1}+\tilde{y}_{D} \kappa_{2}\right), \\
M_{R}=\sqrt{2} y_{M} v_{R},
\end{gathered}
$$

where $y_{M}$ is a $3 \times 3$ Majorana-type Yukawa coupling matrix. $M_{\nu}$ is diagonalized by a $6 \times 6$ unitary matrix $V: V^{T} M_{\nu} V=\left(M_{\nu}\right)_{\text {diag }}$. This matrix relates neutrino mass and flavor eigenstates: $n_{L}^{\prime}=V^{*} N_{L}$, $n_{R}^{\prime}=V N_{R}$.

Three eigenvalues of $M_{\nu}$ are small (denoted later by $m_{\nu}$ ), of the order-of $M_{D}^{2} / M_{R}$, and correspond to the light neutrinos of the SM. For $M_{R}$ in the multi-TeV range, identifying $M_{D}$ with the charged lepton mass clearly violates the $95 \%$ C. L. limit $\sum m_{\nu}<0.7 \mathrm{eV}$ [30] from WMAP. Indeed, for $M_{R} \sim 1 \mathrm{TeV}$, one would need $M_{D} \sim$ $1 \mathrm{MeV}$ to obtain the correct magnitude for the neutrino masses. For the first generation $M_{l}^{e}=0.511 \mathrm{MeV}$, and thus setting $M_{D} \sim M_{l}$ is acceptable. However, for the second and third generations $M_{l}$ is, respectively, $106 \mathrm{MeV}$ and $1.77 \mathrm{GeV}$. Therefore, one would need $M_{D}^{\mu} / M_{l}^{\mu} \lesssim 10^{-2}$ and $M_{D}^{\tau} / M_{l}^{\tau} \lesssim 10^{-3}$. The last value, $10^{-3}$, can be taken as a measure of the fine-tuning needed to accommodate realistic neutrino masses in the LRSM with $M_{R} \sim 1 \mathrm{TeV}$. Such fine-tuning can be achieved, however, with appropriate choices of the Yukawa couplings $y_{D}$ and $\tilde{y}_{D}$.

The remaining three eigenstates are predominantly right-handed with mass $M_{n} \sim M_{R} \sim y_{M} v_{R \sim}^{>} 1 \mathrm{TeV}$, since we assume $y_{M} \sim \mathcal{O}(1)$. The amount of heavy-light mixing of the neutrino sector is set by the ratio $\epsilon \sim$ $M_{D} / M_{R} \sim y_{D} \kappa / y_{M} v_{R} \ll 1$. As discussed below, we will expand our results in $\epsilon$ and $\left(\kappa / v_{R}\right)^{2}$, and retain leading nonvanishing order. Typical values for these expansion parameters are $\left(\kappa / v_{R}\right)^{2} \sim 10^{-2}-10^{-4}$ and $\epsilon \sim 10^{-6}$, resulting from taking $v_{R} \sim 1-10 \mathrm{TeV}, y_{M} \sim O(1)$, and requiring light neutrino masses to be consistent with experimental limits. Since we only consider the leading order terms, the unequal values of the parameters $\epsilon$ and $\left(\kappa / v_{R}\right)^{2}$ are of no concern.

The LFV couplings of leptons to gauge and Higgs bosons are conveniently parametrized in terms of two $6 \times 3$ matrices [28]

$$
K_{L}=V_{L}^{\nu \dagger} V_{L}^{l}, \quad K_{R}=V_{R}^{\nu \dagger} V_{R}^{l}, \quad V=\left(\begin{array}{c}
V_{L}^{\nu *} \\
V_{R}^{\nu}
\end{array}\right) .
$$

At the leading order in $\epsilon$, the upper $3 \times 3$ block of $K_{L}$ and the lower $3 \times 3$ block of $K_{R}$, respectively, describe flavor mixing in the light and heavy neutrino sectors. They are analogous to the CKM matrix which appears in the quark sector of the SM, and satisfy unitarity conditions up to corrections of order $\epsilon^{2}$. In particular, the upper $3 \times 3$ block of $K_{L}$ is the familiar mixing matrix for light neutrinos [31]. As observed in the introduction, contributions involving light neutrinos (and $K_{L}$ ) to any LFV process are GIM suppressed relative to those involving heavy neutrinos. Therefore, the leading contributions to the LFV processes we consider depend on the masses and flavor mixing of heavy neutrinos only.

\section{Gauge Fields}

The charged gauge bosons acquire the following mass matrix

$$
\tilde{M}_{W}^{2}=\frac{g^{2}}{4}\left(\begin{array}{cc}
\kappa_{+}^{2} & -2 \kappa_{1} \kappa_{2} \\
-2 \kappa_{1} \kappa_{2} & \kappa_{+}^{2}+2 v_{R}^{2}
\end{array}\right),
$$

which is diagonalized via the mixing angle $\xi=$ $-\tan ^{-1}\left(2 \kappa_{1} \kappa_{2} / v_{R}^{2}\right) / 2$ with the eigenvalues $M_{W_{1,2}}^{2}=$ $g^{2}\left(\kappa_{+}^{2}+v_{R}^{2} \mp \sqrt{v_{R}^{4}+4 \kappa_{1}^{2} \kappa_{2}^{2}}\right) / 4$. Here $\kappa_{+}=\sqrt{\kappa_{1}^{2}+\kappa_{2}^{2}}$, and the mass eigenstates are related to the gauge eigenstates by

$$
\begin{aligned}
& W_{L}=\cos \xi W_{1}+\sin \xi W_{2} \\
& W_{R}=-\sin \xi W_{1}+\cos \xi W_{2} .
\end{aligned}
$$


The mass matrix for the neutral gauge bosons is

$$
\tilde{M}_{0}^{2}=\frac{1}{2}\left(\begin{array}{ccc}
\frac{g^{2}}{2} \kappa_{+}^{2} & -\frac{g^{2}}{2} \kappa_{+}^{2} & 0 \\
-\frac{g^{2}}{2} \kappa_{+}^{2} & \frac{g^{2}}{2}\left(\kappa_{+}^{2}+4 v_{R}^{2}\right) & -2 g g^{\prime} v_{R}^{2} \\
0 & -2 g g^{\prime} v_{R}^{2} & 2 g^{\prime 2} v_{R}^{2}
\end{array}\right) .
$$

It has the following nonzero eigenvalues (the third one is vanishing)

$$
M_{Z_{1,2}}^{2}=\frac{1}{4}\left[g^{2} \kappa_{+}^{2}+2 v_{R}^{2}\left(g^{2}+g^{\prime 2}\right) \mp \sqrt{\left[g^{2} \kappa_{+}^{2}+2 v_{R}^{2}\left(g^{2}+g^{\prime 2}\right)\right]^{2}-4 g^{2}\left(g^{2}+2 g^{\prime 2}\right) \kappa_{+}^{2} v_{R}^{2}}\right]
$$

The explicit form of the unitary matrix that diagonalizes $\tilde{M}_{0}^{2}$ is given in Ref. [28]. Here, we only list the expression for the $Z_{1}-Z_{2}$ mixing angle:

$$
\phi=-\frac{1}{2} \sin ^{-1} \frac{g^{2} \kappa_{+}^{2} \sqrt{\cos 2 \theta_{W}}}{2 c_{W}^{2}\left(M_{Z_{2}}^{2}-M_{Z_{1}}^{2}\right)}
$$

where $\theta_{W}$ is the weak mixing angle, and $c_{W}\left(s_{W}\right)$ is $\cos \theta_{W}\left(\sin \theta_{W}\right)$ (we use this abbreviation throughout). In this paper we work in the regime where $\phi, \xi \ll 1^{3}$, since both are $\mathcal{O}\left[\left(\kappa / v_{R}\right)^{2}\right]$. Note that because $y_{D} \ll y_{M}$ we have $\epsilon<\phi, \xi$.

\section{Higgs Fields}

With the Higgs sector described above, there are six neutral and four charged physical Higgs bosons [28]. At leading order in $\epsilon$, however, the neutral Higgs bosons do not contribute to the LFV processes involving charged leptons in the external states, and we do not consider them in the following. Two of the remaining bosons, $H_{1,2}^{+}$, are singly charged, with masses $M_{H_{1,2}}$. The last two bosons, $\delta_{L, R}^{++}$, are doubly-charged, with masses $M_{\delta_{L, R}^{++}}$. Masses of the Higgs bosons depend on a number of parameters in the Higgs potential, with the natural scale $M_{H} \sim M_{\delta} \sim$ $v_{R}[19,28]$.

\section{B. Lepton Interactions}

The LFV interactions of leptons with gauge $\left(W_{2}\right)$, singly and doubly-charged bosons are given by the following lagrangian densities:

$$
\begin{gathered}
\mathcal{L}_{C C}=\frac{g}{\sqrt{2}}\left\{N\left[\gamma^{\mu} P_{R}\left(K_{R}\right)\right] l \cdot W_{2 \mu}^{+}\right. \\
\left.+\bar{l}\left[\gamma^{\mu} P_{R}\left(K_{R}^{\dagger}\right)\right] N \cdot W_{2 \mu}^{-}\right\} \\
\mathcal{L}_{H_{1}}=\frac{g}{\sqrt{2}}\left[H_{1}^{+} \bar{N}\left(\tilde{h} P_{L}\right) l+H_{1}^{-} \bar{l}\left(\tilde{h}^{\dagger} P_{R}\right) N\right] \\
\mathcal{L}_{\delta_{L, R}^{ \pm \pm}}=\frac{g}{2}\left[\delta_{L, R}^{++} \bar{l}^{c}\left(h_{L, R} P_{L, R}\right) l+\delta_{L, R}^{-} \bar{l}\left(h_{L, R}^{\dagger} P_{R, L}\right) l^{c}\right],
\end{gathered}
$$

\footnotetext{
${ }^{3}$ The experimental limits on the gauge boson mixing angles are $|\xi|<3 \times 10^{-3}$ and $|\phi|<1.8 \times 10^{-3}[31]$
}

where $P_{L, R}=\left(1 \mp \gamma_{5}\right) / 2, N=N_{L}+N_{R}=N^{c}, l=l_{L}+$ $l_{R}$ and where we have neglected $\mathcal{O}(\xi)$ terms. With our choice of the Higgs sector, it follows [19] that the $3 \times 3$ matrix couplings $h_{L}$ and $h_{R}$ can be identical (manifest left-right symmetry) or can have components differing by a sign (quasimanifest left-right symmetry). In the manifest left-right symmetry case one finds

$$
h_{L}=h_{R}=K_{R}^{T} \frac{M_{\nu}^{\mathrm{diag}}}{M_{W_{2}}} K_{R} \equiv h \quad \tilde{h}=K_{L}^{*} h_{L}
$$

Note that it is $K_{R}^{T}$, not $K_{R}^{\dagger}$ that appears in the definition of $h$. Because $K_{R}$ may contain Majorana phases, $h$ is not necessarily proportional to the unit matrix even if all heavy neutrinos are degenerate. At leading order in $\epsilon$ one has

$$
\begin{gathered}
h_{i j}=\sum_{n=\text { heavy }}\left(K_{R}\right)_{n i}\left(K_{R}\right)_{n j} \sqrt{x_{n}}, \\
\left(h^{\dagger} h\right)_{e \mu}=\left(\tilde{h}^{\dagger} \tilde{h}\right)_{e \mu}=\sum_{n=\text { heavy }} x_{n}\left(K_{R}^{\dagger}\right)_{e n}\left(K_{R}\right)_{n \mu} \equiv g_{\text {lfv }},
\end{gathered}
$$

$$
x_{n}=\left(\frac{M_{n}}{M_{W_{2}}}\right)^{2} \text {, }
$$

where the sum is over the heavy neutrinos only. Equation (25) relates the lepton-gauge boson couplings to the lepton-Higgs triplet couplings. We emphasize that it is specific to left-right symmetric models, and plays a central role in phenomenological applications. Generalization to the quasimanifest left-right symmetry case is trivial and we have explicitly checked that a possible relative sign between $h_{L}$ and $h_{R}$ has no observable consequences in LFV processes. Finally, note that for degenerate heavy neutrinos, i.e., $x_{n}=$ const, one has $g_{\text {lfv }}=\mathcal{O}\left(\epsilon^{2}\right)$ due to the approximate unitarity of the lower $3 \times 3$ block of $K_{R}$. Thus $g_{\text {lfv }}$ depends only on the mass square differences of the heavy neutrinos. The same is not true for the individual $h_{i j} \mathrm{~s}$. 


\section{CALCULATION}

Within the LRSM we performed a complete calculation of the LFV muon processes $\mu \rightarrow e \gamma, \mu \rightarrow e$ conversion in nuclei, and $\mu \rightarrow 3 e$ to leading order in the expansion parameters $\left(\kappa / v_{R}\right)^{2}$ and $\epsilon \sim M_{D} / M_{R}$. Diagrammatic contributions fall into three classes, schematically shown in Fig. 1. Given the lagrangian in the physical fields basis [28], we have first identified all diagrams contributing to leading order in $\left(\kappa / v_{R}\right)^{2}$ and $M_{D} / M_{R}$. We have then calculated the LFV vertices $\mu \rightarrow e \gamma^{*}, \mu \rightarrow e Z_{1}^{*}$, and $\mu \rightarrow e Z_{2}^{*}$ [Fig. 1(a) ]. Finally, we have combined the LFV effective vertices with the $\bar{q} q \gamma^{*}, \bar{q} q Z_{1,2}^{*}$ interactions, and relevant box-type [Fig. 1(b) ] and tree-level diagrams [Fig. 1(c) ] to obtain the effective lagrangian for $\mu \rightarrow e$ conversion and $\mu \rightarrow 3 e$. Our calculation and main results are described in this section, and some technical details are given in the appendices $\mathrm{A}$ and $\mathrm{B}$.

\section{A. Identifying the Leading Contributions}

In the LRSM at low-energy all effects of the righthanded sector are suppressed by powers of $\kappa / v_{R}$, as a consequence of the decoupling theorem [26]. In our analysis we keep only the leading contributions in the expansion parameters $\left(\kappa / v_{R}\right)^{2}$ and $\epsilon$. Throughout, we work in 't Hooft-Feynman gauge. Our findings can be summarized as follows:

(i) The $\mu \rightarrow e \gamma^{*}$ vertex receives its leading contributions at order $\epsilon^{0},\left(\kappa / v_{R}\right)^{0}$. In accordance with electromagnetic gauge invariance, however, the momentum-independent piece of the amplitude vanishes, so that the resulting vertex

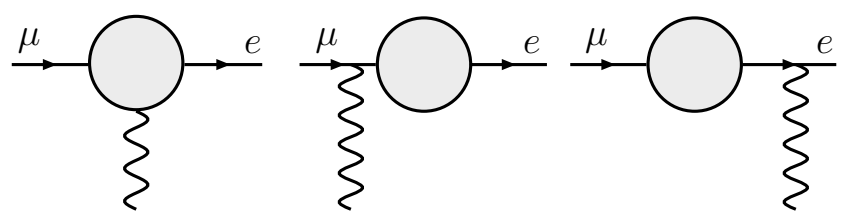

a)

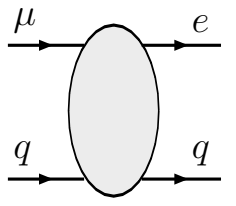

b)

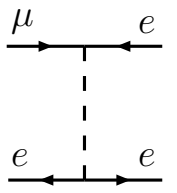

c)
FIG. 1. Diagrams contributing to $\mu \rightarrow e \gamma, \mu \rightarrow e$ conversion in nuclei, and $\mu \rightarrow 3 e$. The wavy lines represent neutral gauge bosons $\left(\gamma\right.$ or $Z_{1,2}$ ). $\mu \rightarrow e \gamma$ is described by class (a). $\mu \rightarrow$ $e$ conversion is described by class (a) (attaching a quark line to the neutral gauge boson) and class (b) (with two external quark legs). $\mu \rightarrow 3 e$ receives in principle contributions from classes (a) (attaching an electron line to the gauge boson), (b), and (c). function is actually of order $q^{2} / v_{R}^{2}, q$ being the momentum transfer. Our expression is fully gauge invariant and respects the decoupling theorem. Note that when this vertex is inserted into the $\mu \rightarrow e$ conversion amplitude, the $q^{2}$ in the vertex cancels the $1 / q^{2}$ of the photon propagator, leaving a contact interaction that scales as $\sim 1 / v_{R}^{2}$.

(ii) The $\mu \rightarrow e Z_{2}^{*}$ vertex is again not suppressed by powers of $\epsilon$ or $\left(\kappa / v_{R}\right)$, and we only keep the momentum-independent component. This result does not contradict the decoupling theorem, as one of the external states belongs to the heavy sector of the theory. Since $q^{2} \ll M_{Z_{2}}^{2}$, the contribution from this vertex to the $\mu \rightarrow e$ conversion amplitude goes as $1 / v_{R}^{2}$.

(iii) The $\mu \rightarrow e Z_{1}^{*}$ vertex nominally receives its leading contributions at order $\epsilon^{0},\left(\kappa / v_{R}\right)^{0}$. However, the momentum-independent part of this class of diagrams sums to zero, in accordance with the decoupling theorem. We find that the leading nonvanishing contribution is $\mathcal{O}\left[\left(\kappa / v_{R}\right)^{2}\right]$. Consequently, the contribution from this vertex to the conversion amplitude is also $\sim 1 / v_{R}^{2}$.

For the kinematics of the LFV decays considered here, the momentum dependent contributions to the $\mu \rightarrow e Z_{1,2}^{*}$ vertices are highly suppressed and can be neglected.

\section{B. Effective Vertices}

The $\mu \rightarrow e$ LFV vertices can be expressed in terms of known couplings and form factors $F_{L, R}^{(i)}, A_{L, R}$ as follows,

$$
\begin{gathered}
L_{\mu}^{\left(Z_{1}\right)=}=\frac{e G_{F} M_{W_{1}}^{2}}{\sqrt{2}(4 \pi)^{2}} \frac{1}{s_{W} c_{W}} \bar{e} \gamma_{\mu}\left(F_{L}^{(1)} P_{L}+F_{R}^{(1)} P_{R}\right) \mu \\
L_{\mu}^{\left(Z_{2}\right)=}=\frac{e G_{F} M_{W_{1}}^{2}}{\sqrt{2}(4 \pi)^{2}} \frac{1}{s_{W} c_{W} \sqrt{\cos 2 \theta_{W}}} \bar{e} \gamma_{\mu} \\
\quad \times\left(F_{L}^{(2)} P_{L}+F_{R}^{(2)} P_{R}\right) \mu \\
L_{\mu}^{(\gamma)}=\frac{e G_{F}}{\sqrt{2}(4 \pi)^{2}} \bar{e}\left\{\left(q^{2} \gamma_{\mu}-/ \gamma \cdot q q_{\mu}\right)\left(F_{L}^{(\gamma)} P_{L}+F_{R}^{(\gamma)} P_{R}\right)\right. \\
\left.-i 8(4 \pi)^{2} m_{\mu} \sigma_{\mu \nu} q^{\nu}\left(A_{L} P_{L}+A_{R} P_{R}\right)\right\} \mu
\end{gathered}
$$

where $q=p_{e}-p_{\mu}, \sigma_{\mu \nu}=\frac{i}{2}\left[\gamma_{\mu}, \gamma_{\nu}\right]$. The $\mu e \gamma^{*}$ effective vertex has both "anapole" $\left(F_{L, R}^{(\gamma)}\right)$ and dipole $\left(A_{L, R}\right)$ terms ${ }^{4}$. Only the dipole terms contribute to the on-shell

\footnotetext{
${ }^{4}$ The first term in Eq. (29) involves a coupling of the flavorviolating lepton current to the electromagnetic current rather than to a field associated with the corresponding vector potential. Zeldovich referred to this interaction as an anapole coupling [32].
} 
decay $\mu \rightarrow e \gamma$, while both anapole and dipole contribute to $\mu$ to $e$ conversion in nuclei.

The $\mu \rightarrow e$ effective vertices receive contributions from the one-particle-irreducible diagrams depicted in Fig. 2, as well as from external-leg corrections. The vertex corrections can be grouped into three classes: (i) gauge contributions (including unphysical Higgs exchange), (ii) singly charged physical Higgs contribu tions, and (iii) doubly-charged Higgs contributions. Power counting implies that only certain combinations of gauge bosons, neutrinos, and Higgs particles contribute to leading order in $\left(\kappa / v_{R}\right)^{2}$ and $\epsilon$. The relevant intermediate states are indicated diagram by diagram in Table I.

\section{1. $\mu e Z_{1}^{*}$ Vertex}

In this case the leading diagrams involving triplet Higgs (singly and doubly-charged) sum to zero, and the main effect stems from gauge contributions. When working in 't Hooft-Feynman gauge, one needs to include the

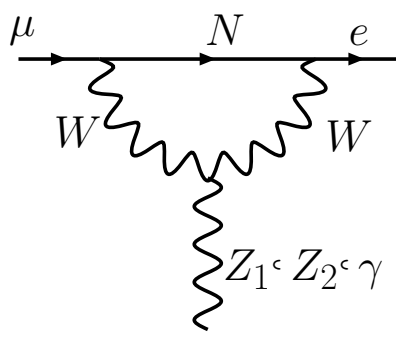

a)

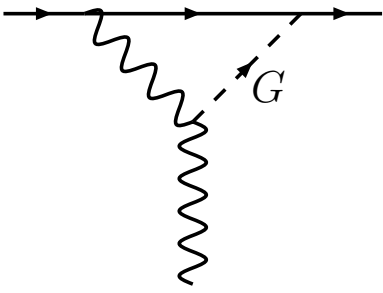

c)

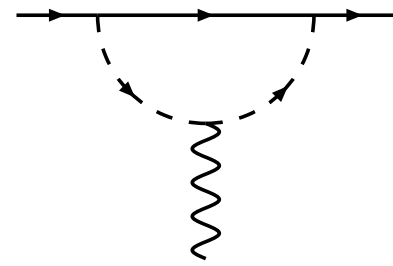

e)

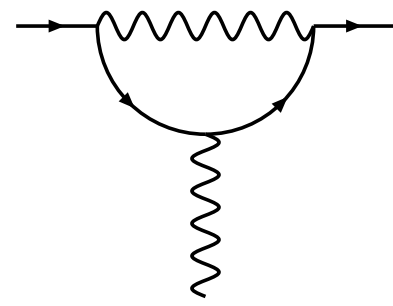

b)

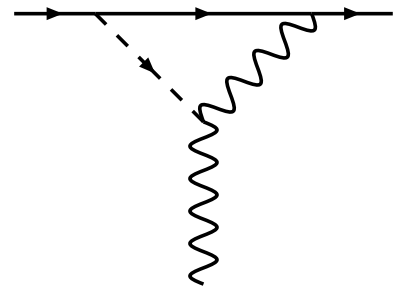

d)

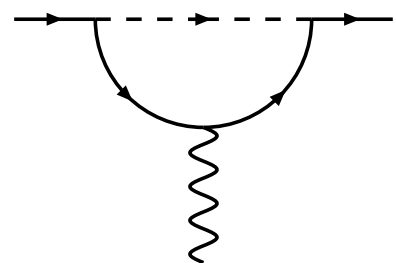

f)
FIG. 2. Basic topologies for the one-particle-irreducible contributions to the $\mu e \gamma^{*}, \mu e Z_{1}^{*}$, and $\mu e Z_{2}^{*}$ effective vertices (detailed version of Fig. 1(a)]. Wavy lines represent gauge bosons, dashed lines represent scalars (physical or unphysical), full lines represent leptons (charged or neutral). The internal particles contributing at leading order to each topology are listed in Table I. effect of unphysical Higgs exchange, and their mixing with other physical and unphysical scalars of the theory (terms proportional to $\eta_{1}$ and $\eta_{2}$ below). In terms of the heavy neutrino masses $\left(M_{n}\right)$, mixing matrix $K_{R}$, and the ratios $x_{n}=\left(M_{n} / M_{W_{2}}\right)^{2}, \quad y_{n}=\left(M_{n} / M_{W_{1}}\right)^{2}, \quad z_{n}=$ $\left(M_{n} / M_{H_{2}}\right)^{2}$, the resulting form factors have the following structure:

$$
\begin{aligned}
F_{R}^{(1)}= & \sum_{n=\text { heavy }}\left(K_{R}^{\dagger}\right)_{e n}\left(K_{R}\right)_{n \mu} \\
& \times\left[\eta_{0} S_{1}\left(x_{n}\right)+2 \eta_{1} D_{1}\left(x_{n}, y_{n}\right)+\eta_{2} D_{1}\left(x_{n}, z_{n}\right)\right],
\end{aligned}
$$

$$
F_{L}^{(1)}=\mathcal{O}\left(\frac{m_{\nu}^{2}}{M_{W_{1}}^{2}}\right) \ll F_{R}^{(1)},
$$

where $\left(\kappa_{-}^{2}=\kappa_{1}^{2}-\kappa_{2}^{2}\right)$

$$
\begin{gathered}
\eta_{0}=-\frac{\sin \phi c_{W}^{2}}{\sqrt{\cos 2 \theta_{W}}} \simeq \frac{M_{Z_{1}}^{2} c_{W}^{2}}{M_{Z_{2}}^{2}-M_{Z_{1}}^{2}} \simeq \frac{1-2 s_{W}^{2}}{2 c_{W}^{2}} \frac{M_{W_{1}}^{2}}{M_{W_{2}}^{2}}, \\
\eta_{1}=\left(\frac{\kappa_{1} \kappa_{2}}{\kappa_{+} v_{R}}\right)^{2} \simeq \frac{1}{2}\left(\frac{M_{W_{2}}}{M_{W_{1}}} \sin \xi\right)^{2} \leq \frac{1}{2}\left(\frac{M_{W_{1}}}{M_{W_{2}}}\right)^{2} \\
\eta_{2}=\left(\frac{\kappa_{-}^{2}}{\sqrt{2} \kappa_{+} v_{R}}\right)^{2} \leq\left(\frac{M_{W_{1}}}{M_{W_{2}}}\right)^{2}
\end{gathered}
$$

and the functions $S_{1}(x), D_{1}(x, y)$ are defined in Appendix A.

\section{2. $\mu e Z_{2}^{*}$ Vertex}

As in the previous case, the leading term arises from gauge-lepton interactions, and the leading physical Higgs effects cancel out. With the notation established above, we find:

$$
\begin{gathered}
F_{R}^{(2)}=c_{W}^{2} \sum_{n=\text { heavy }}\left(K_{R}^{\dagger}\right)_{e n}\left(K_{R}\right)_{n \mu} S_{1}\left(x_{n}\right), \\
F_{L}^{(2)}=\mathcal{O}\left(\frac{m_{\nu}^{2}}{M_{W_{1}}^{2}}\right) \ll F_{R}^{(2)} .
\end{gathered}
$$

\section{3. $\mu e \gamma^{*}$ Vertex}

Both anapole and dipole transition form factors receive nonvanishing leading contribution from gauge diagrams and exchange of singly and doubly-charged triplet Higgs particles. Neglecting charged fermion masses (see Appendix B) and using Eq. (25), the various amplitudes read: 
TABLE I. Intermediate states contributing at leading order in $\left(\kappa / v_{R}\right)^{2}$ and $\epsilon$ to $\mu e \gamma^{*}, \mu e Z_{1}^{*}$, and $\mu e Z_{2}^{*}$ effective vertices in 't Hooft-Feynman gauge. For each topology in Fig. 2 we list the intermediate states as they appear starting from the muon vertex and following the loop counter-clockwise. Neutrinos are denoted by $N_{\mathrm{h}}$ (heavy) and $N_{1}$ (light). $G_{1,2}$ denote the unphysical Higgs fields associated with the longitudinal polarization of the gauge bosons $W_{1,2}$.

\begin{tabular}{|c|c|c|c|c|c|c|}
\hline & a) & b) & c) & d) & e) & f) \\
\hline$\gamma$ & $W_{2}, W_{2}, N_{\mathrm{h}}$ & & $W_{2}, G_{2}, N_{\mathrm{h}}$ & $G_{2}, W_{2}, N_{\mathrm{h}}$ & $\begin{array}{c}G_{2}, G_{2}, N_{\mathrm{h}} \\
H_{1}, H_{1}, N_{1} \\
\delta_{L, \frac{ \pm}{R}}^{ \pm}, \delta_{L, \frac{ \pm}{R}}^{ \pm}, l_{i}\end{array}$ & $l_{i}, l_{i}, \delta_{L, \frac{ \pm}{R}}^{ \pm}$ \\
\hline$Z_{1}$ & $W_{2}, W_{2}, N_{\mathrm{h}}$ & $N_{\mathrm{h}}, N_{\mathrm{h}}, W_{2}$ & $\begin{array}{l}W_{2}, G_{2}, N_{\mathrm{h}} \\
W_{2}, H_{2}, N_{\mathrm{h}} \\
W_{2}, G_{1}, N_{\mathrm{h}}\end{array}$ & $\begin{array}{l}G_{2}, W_{2}, N_{\mathrm{h}} \\
H_{2}, W_{2}, N_{\mathrm{h}} \\
G_{1}, W_{2}, N_{\mathrm{h}}\end{array}$ & $\begin{array}{c}G_{2}, G_{2}, N_{\mathrm{h}} \\
H_{2}, H_{2}, N_{\mathrm{h}} ; G_{2}, H_{2}, N_{\mathrm{h}} ; H_{2}, G_{2}, N_{\mathrm{h}} \\
G_{1}, G_{1}, N_{\mathrm{h}} ; G_{1}, G_{2}, N_{\mathrm{h}} ; G_{2}, G_{1}, N_{\mathrm{h}}\end{array}$ & $N_{\mathrm{h}}, N_{\mathrm{h}}, G_{2}$ \\
\hline$Z_{2}$ & $W_{2}, W_{2}, N_{\mathrm{h}}$ & $N_{\mathrm{h}}, N_{\mathrm{h}}, W_{2}$ & $W_{2}, G_{2}, N_{\mathrm{h}}$ & $G_{2}, W_{2}, N_{\mathrm{h}}$ & $G_{2}, G_{2}, N_{\mathrm{h}}$ & $N_{i}, N_{i}, G_{2}$ \\
\hline
\end{tabular}

$$
\begin{gathered}
F_{R}^{(\gamma)}=\sum_{n=\text { heavy }}\left(K_{R}^{\dagger}\right)_{e n}\left(K_{R}\right)_{n \mu} \\
\times\left[\frac{M_{W_{1}}^{2}}{M_{W_{2}}^{2}} S_{2}\left(x_{n}\right)-x_{n} \frac{8}{3} \frac{M_{W_{1}}^{2}}{M_{\delta_{R}^{++}}^{2}} \log \left(\frac{-q^{2}}{M_{\delta_{R}^{++}}^{2}}\right)\right], \\
F_{L}^{(\gamma)}=\sum_{n=\text { heavy }}\left(K_{R}^{\dagger}\right)_{e n}\left(K_{R}\right)_{n \mu} x_{n} \\
\times\left[-\frac{8}{3} \frac{M_{W_{1}}^{2}}{M_{\delta_{L}^{++}}^{2}} \log \left(\frac{-q^{2}}{M_{\delta_{L}^{++}}^{2}}\right)-\frac{2}{9} \frac{M_{W_{1}}^{2}}{M_{H_{1}^{+}}^{2}}\right], \\
A_{L}=\frac{1}{16 \pi^{2}} \sum_{n=\text { heavy }}\left(K_{R}^{\dagger}\right)_{e n}\left(K_{R}\right)_{n \mu} \\
\times\left[\frac{M_{W_{1}}^{2}}{M_{W_{2}}^{2}} S_{3}\left(x_{n}\right)-\frac{x_{n}}{3} \frac{M_{W_{1}}^{2}}{M_{\delta_{R}^{++}}^{2}}\right] \\
\quad \times\left[-\frac{1}{3} \frac{M_{W_{1}}^{2}}{M_{\delta_{L}^{++}}^{2}}-\frac{1}{24} \frac{M_{W_{1}}^{2}}{M_{H_{1}^{+}}^{2}}\right] \\
A_{R}=\frac{1}{16 \pi^{2}} \sum_{n=\text { heavy }}\left(K_{R}^{\dagger}\right)_{e n}\left(K_{R}\right)_{n \mu} x_{n} \\
\times
\end{gathered}
$$

The functions $S_{2,3}(x)$ are given explicitly in Appendix A. The most important feature of these results is the logarithmic enhancement $\left(q^{2} \simeq-m_{\mu}^{2}\right)$ of the anapole transition form factors, arising from the doubly-charged triplet Higgs diagrams. This implies that in the left-right symmetry framework, $\mu \rightarrow e$ conversion in nuclei is as strong probe of LFV as $\mu \rightarrow e \gamma$ since its amplitude is logarithmically-enhanced, and thus compensates for the extra factor of $\sim \alpha$. This effect was pointed out for a larger class of models in Ref. [25] within an effective field theory approach. Its consequences within the LRSM will be discussed in the next section in detail.

In Appendix B we report full expressions for the $\mu e \gamma^{*}$ form factors (including charged fermion masses) in terms of $h, \tilde{h}$ (i.e., without using Eq. (25)).

\section{Effective Lagrangian for $\mu \rightarrow \boldsymbol{e}$ Conversion}

The effective Lagrangian for $\mu \rightarrow e$ conversion receives contributions from (i) tree-level exchange of heavy neutral Higgs states; (ii) box diagrams depicted in Fig. 3; (iii) LFV effective vertices, with the gauge boson attached to a quark line (the relevant quark-gauge couplings are summarized in Table II). Inspection of the neutral Higgs couplings implies that the ratio of effective couplings $g_{\text {tree }}^{\text {eff }}$ and $g_{\text {loop }}^{\text {eff }}$ generated by tree-level Higgs exchange and loop corrections, respectively, scales as $g_{\text {tree }}^{\text {eff }} / g_{\text {loop }}^{\text {eff }} \sim$ $\left(y_{D}\right)^{2} /(\alpha / 4 \pi) \ll 1$. Therefore, we safely neglect the Yukawa suppressed tree-level diagrams.

After casting the $\mu e Z_{1,2}^{*}$ vertices and the $\mu e \gamma^{*}$ anapole terms in the form of a current-current interaction, the effective lagrangian can be written as in Refs. [33,34]:

$$
\begin{aligned}
\mathcal{L}_{\mu \rightarrow e}= & -\frac{4 G_{F} e}{\sqrt{2}} m_{\mu} \bar{e} \sigma_{\mu \nu}\left(A_{L} P_{L}+A_{R} P_{R}\right) \mu \cdot F^{\mu \nu} \\
& -\frac{G_{F}}{\sqrt{2}} \sum_{q}\left\{\bar{e} \gamma_{\mu}\left[g_{L V}(q) P_{L}+g_{R V}(q) P_{R}\right] \mu\right. \\
& \otimes \bar{q} \gamma^{\mu} q+\bar{e} \gamma_{\mu}\left[g_{L A}(q) P_{L}+g_{R A}(q) P_{R}\right] \mu \\
& \left.\otimes \bar{q} \gamma^{\mu} \gamma^{5} q\right\}+ \text { h.c. },
\end{aligned}
$$

where $F^{\mu \nu}$ has to be understood as the classical field produced by the nucleus. In terms of the box contribution $\left(S_{4}(x)\right.$ is defined in Appendix A)

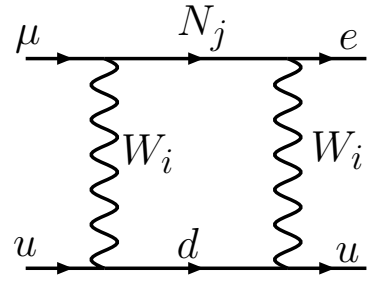

a)

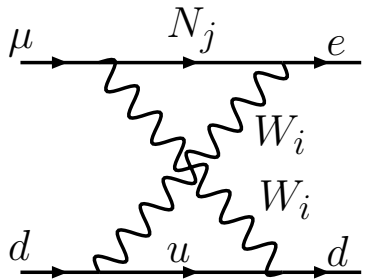

b)
FIG. 3. Box diagrams contributing to $F_{R}^{(\mathrm{B})}$ [Fig. 1(b)]. 
TABLE II. Vector and Axial-Vector couplings of $u$ and $d$ quarks to $Z_{1}, Z_{2}$, and $\gamma$. We list for completeness the effective Vector and Axial-Vector couplings induced by box diagrams of Fig. 3.

\begin{tabular}{|c|c|c|c|}
\hline $\begin{array}{l}Z_{1} \\
v_{u}^{(1)}=1-\frac{8}{3} s_{W}^{2} \\
a_{u}^{(1)}=1\end{array}$ & $\begin{array}{c}Z_{2} \\
v_{u}^{(2)}=1-\frac{8}{3} s_{W}^{2} \\
a_{u}^{(2)}=-1+2 s_{W}^{2}\end{array}$ & $\boldsymbol{v}_{u}^{(\gamma) \stackrel{\gamma}{=} \frac{2}{3}}$ & $\begin{array}{c}\mathrm{BOX} \\
v_{u}^{(\mathrm{B})}=1 \\
a_{u}^{(\mathrm{B})}=-1\end{array}$ \\
\hline $\begin{array}{l}v_{d}^{(1)}=-1+\frac{4}{3} s_{W}^{2} \\
a_{d}^{(1)}=-1\end{array}$ & $\begin{array}{l}v_{d}^{(2)}=-1+\frac{4}{3} s_{W}^{2} \\
a_{d}^{(2)}=1-2 s_{W}^{2}\end{array}$ & $v_{d}^{(\gamma)}=-\frac{1}{3}$ & $\begin{array}{l}v_{d}^{(\mathrm{B})}=-\frac{1}{4} \\
a_{d}^{(\mathrm{B})}=\frac{1}{4}\end{array}$ \\
\hline
\end{tabular}

$$
F_{R}^{(\mathrm{B})}=8 \sum_{n=\text { heavy }}\left(K_{R}^{\dagger}\right)_{e n}\left(K_{R}\right)_{n \mu} S_{4}\left(x_{n}\right),
$$

and the LFV form factors, the couplings $g_{L V, R V}(q)$ are

$$
\begin{gathered}
g_{L V}(q)=-\frac{\alpha}{4 \pi} F_{L}^{(\gamma)} \boldsymbol{v}_{q}^{(\gamma)} \\
g_{R V}(q)=\frac{\alpha}{8 \pi s_{W}^{2}}\left\{-2 \sin ^{2} \theta_{W} F_{R}^{(\gamma)} \boldsymbol{v}_{q}^{(\gamma)}+\frac{1}{2} F_{R}^{(1)} \boldsymbol{v}_{q}^{(1)}\right. \\
\left.+\frac{M_{W_{1}}^{2}}{M_{W_{2}}^{2}} \frac{F_{R}^{(2)} \boldsymbol{v}_{q}^{(2)}}{4 c_{W}^{4}}-\frac{M_{W_{1}}^{2}}{M_{W_{2}}^{2}} F_{R}^{(B)} v_{q}^{(B)}\right\}
\end{gathered}
$$

The expressions for $g_{L A, R A}(q)$ are obtained by replacing $v_{q}^{(i)}$ with $a_{q}^{(i)}$ in $g_{L V, R V}(q)$. We remark that all the contributions to $g_{R V}(q)$ in Eq. (44) enter at leading order $\kappa / v_{R}$, contrary to what appears in earlier calculations $[17,18]$. In Ref. [17] only $F_{R}^{(2)}$ and $F_{R}^{(B)}$ were included, while the authors of Ref. [18] considered only $F_{L, R}^{(1)}$. Both of these previous studies omitted the dominant, logarithmicallyenhanced contributions from $F_{L, R}^{(\gamma)}$. Finally, we note that upon taking matrix elements of $\mathcal{L}_{\mu \rightarrow e}$ in nuclei, the following combinations of $g_{L V, R V}(q)$ become relevant:

$$
\begin{gathered}
\tilde{g}_{L V, R V}^{(p)}=2 g_{L V, R V}(u)+g_{L V, R V}(d), \\
\tilde{g}_{L V, R V}^{(n)}=g_{L V, R V}(u)+2 g_{L V, R V}(d) .
\end{gathered}
$$

\section{Effective Lagrangian for $\mu \rightarrow 3 e$}

The process $\mu \rightarrow 3 e$ can occur in the LRSM through (i) tree-level exchange of doubly-charged Higgses (via the interaction of Eq. (22)); (ii) one-loop effective $\mu \rightarrow e$ vertex, with an electron line attached to the gauge boson; (iii) box diagrams. Barring the unnatural possibility that $M_{\delta_{L, R}^{ \pm \pm}} \gg M_{W_{2}}$, the loop amplitudes (ii) and (iii) are suppressed by the standard $\alpha / \pi$ factor, and therefore in our analysis we disregard them.

Doubly-charged Higgs particles mediate at tree-level also the decays $\tau \rightarrow l_{a} l_{b} \bar{l}_{c}$, with $l_{a, b, c}=\mu, e$. In compact notation, the effective lagrangian for four-lepton pro- cesses is given by:

$$
\mathcal{L}_{\delta}=\frac{g^{2}}{4} h_{i j} h_{k m}^{*}\left[\frac{1}{M_{\delta_{R}^{++}}^{2}}\left(\bar{l}_{i R}^{c} l_{j R}\right)\left(\bar{l}_{k R} l_{m R}^{c}\right)+(L \leftrightarrow R)\right]
$$

\section{ANALYSIS}

Based on the results described in the previous section, we now discuss the phenomenology of lepton flavor violation in muon decays within the LRSM. There are three main objectives of our analysis. First, we shall identify relations between LFV rates that are largely independent of the model parameters, and therefore can be considered as signatures of left-right symmetry broken at the multi$\mathrm{TeV}$ scale. The pattern emerging is remarkably clear, and could be confronted with experimental findings in the next decade: the branching fractions for $\mu \rightarrow e$ conversion and $\mu \rightarrow e \gamma$ are expected to be very similar, and two order-of magnitude smaller than the one for $\mu \rightarrow 3 e$ (with some caveats). Second, we shall study the constraints on heavy neutrino masses and mixings implied by present experimental limits on LFV processes. And third, we shall discuss the impact of future experiments, including collider measurements.

Before describing the details of our analysis let us shortly recall the existing limits on the model parameters of interest to us. Direct searches imply that $M_{W_{2}} \geq$ $786 \mathrm{GeV}$, while singly- and doubly-charged Higgs particles should be heavier than $\sim 100 \mathrm{GeV}$ [31]. Indirect bounds are stronger and require the Higgs masses to be on the $\mathrm{TeV}$ scale. In summary, the existing phenomenology is consistent with the heavy sector masses being generically at the $\mathrm{TeV}$ scale or above. In what follows, we shall explore the consequences of a heavy mass scale being in the range 1-10 TeV, which can be tested in the foreseeable future.

\section{A. Setting the stage}

The quantities of primary interest to us are the branching ratios: 


$$
\begin{gathered}
B_{\mu \rightarrow e \gamma}=\frac{\Gamma(\mu \rightarrow e \gamma)}{\Gamma_{\mu}^{(0)}}, \quad B_{\mu \rightarrow e}^{Z}=\frac{\Gamma_{\mathrm{conv}}^{Z}}{\Gamma_{\mathrm{capt}}^{Z}}, \\
B_{\mu \rightarrow 3 e}=\frac{\Gamma(\mu \rightarrow 3 e)}{\Gamma_{\mu}^{(0)}},
\end{gathered}
$$

where $\Gamma_{\mu}^{(0)}=\left(G_{F}^{2} m_{\mu}^{5}\right) /\left(192 \pi^{3}\right)$, and for the capture rate $\Gamma_{\text {capt }}^{Z}$ we take the experimental values. The expression for the conversion rate $\Gamma_{\text {conv }}^{Z}$ involves the overlap integrals [33]

$$
\begin{aligned}
V^{(p, n)} & =\frac{1}{2 \sqrt{2}} \int_{0}^{\infty} d r r^{2} N^{(p, n)} \rho^{(p, n)}\left(g_{e}^{-} g_{\mu}^{-}+f_{e}^{-} f_{\mu}^{-}\right), \\
D & =-\frac{4 m_{\mu}}{\sqrt{2}} \int_{0}^{\infty} d r r^{2} E(r)\left(g_{e}^{-} f_{\mu}^{-}+f_{e}^{-} g_{\mu}^{-}\right) .
\end{aligned}
$$

Here $N^{(p)}=Z, N^{(n)}=A-Z ; \rho^{(p, n)}$ are proton and neutron densities, $E(r)$ is the electric field generated by protons, and $g_{\mu, e}^{-} f_{\mu, e}^{-}$, are the upper and lower components of the initial bound muon and final continuum electron wavefunctions, obtained by solving the Dirac equation. The overlap integrals have dimension of (mass) $)^{5 / 2}$, and in our study we use the numerical results for them reported in Table I of Ref. [33]. In terms of the form factors calculated above and $D, V^{(n)}, V^{(p)}$, the relevant branching fractions read:

$$
\begin{gathered}
B_{\mu \rightarrow e \gamma}=384 \pi^{2} e^{2}\left(\left|A_{L}\right|^{2}+\left|A_{R}\right|^{2}\right), \\
B_{\mu \rightarrow e}=\frac{2 G_{F}^{2}}{\Gamma_{\text {capt }}}\left(\left|A_{R}^{*} D+\tilde{g}_{L V}^{(p)} V^{(p)}+\tilde{g}_{L V}^{(n)} V^{(n)}\right|^{2}\right. \\
\left.+\left|A_{L}^{*} D+\tilde{g}_{R V}^{(p)} V^{(p)}+\tilde{g}_{R V}^{(n)} V^{(n)}\right|^{2}\right), \\
B_{\mu \rightarrow 3 e}=\frac{1}{2}\left|h_{\mu e} h_{e e}^{*}\right|^{2}\left(\frac{M_{W_{1}}^{4}}{M_{\delta_{L}^{++}}^{4}}+\frac{M_{W_{1}}^{4}}{M_{\delta_{R}^{++}}^{4}}\right) .
\end{gathered}
$$

While $B_{\mu \rightarrow 3 e}$ has a relatively simple structure, in general $B_{\mu \rightarrow e \gamma}$ and $B_{\mu \rightarrow e}$ depend on a large number of unknown model parameters. However, under the rather natural assumption of a "commensurate mass spectrum" for the heavy sector of the model (i.e., $M_{W_{2}} \sim M_{\delta_{R}^{++}} \sim$ $\left.M_{\delta_{L}^{++}} \sim M_{H^{+}}\right)$, the problem becomes more tractable. Specifically, if $M_{W_{2}}, M_{\delta_{R}^{++}}, M_{\delta_{L}^{++}}, M_{H^{+}}$, and the heavy neutrino masses $M_{n}$ are all of the same order-of magnitude (in practice we shall assume $0.2_{\sim}^{<} M_{i} / M_{j \sim}^{<} 5$ for each pair of masses), the amplitudes for $\mu \rightarrow e$ and $\mu \rightarrow e \gamma$ become approximately proportional to $g_{\text {lfv }}$, defined in Eq. (25). This is based on the following observations:

(i) Doubly-charged Higgs contributions to the couplings $A_{L, R}, g_{L V}(q)$ and $g_{R V}(q)$ are linear in $x_{n}$ (hence proportional to $g_{\text {lfv }}$ ), and are sizable (the anapole transition form factor receives a large logarithmic enhancement). (ii) Gauge contributions depend on $x_{n}$ through the functions $S_{i}(x)$. These terms always represent a small correction to the Higgs contribution because

$$
\begin{aligned}
& \text { (a): }\left|S_{i}(x)\right| \ll x \frac{8}{3} \log \frac{M_{\delta^{++}}^{2}}{m_{\mu}^{2}} ; \\
& \text { (b): }\left|S_{i}^{\prime}(x)\right| \ll \frac{8}{3} \log \frac{M_{\delta^{++}}^{2}}{m_{\mu}^{2}} ;
\end{aligned}
$$

within the region $(0.2)^{2} \leq x \leq 3$, where the lower limit follows from our assumption of commensurate spectrum and the upper limit from the vacuum stability condition $[35,36] .^{5}$

Condition (a) ensures that gauge terms are small in the case of nondegenerate heavy neutrinos, while condition (b) suppresses them in the case of nearly degenerate neutrinos. In what follows we account for the small gauge-induced contributions to the various couplings by expanding the $S_{i}(x)$ around $\bar{x}=1.5$, and keeping only the linear term. We have checked that the residual dependence on the expansion point $\bar{x}$ is small, and does not affect our discussion and results in a significant way.

The above considerations about the relevance of $g_{\text {lfv }}$ remain true even in the unnatural limit $M_{\delta_{L, R}^{++}} \ll M_{W_{2}}$, but become invalid in the opposite limit $M_{\delta_{L, R}^{++}} \gg M_{W_{2}}$, as for $M_{\delta_{L, R}^{++}} \sim 10 M_{W_{2}}$ the Higgs mass suppression compensates the logarithmic enhancement. Such unnatural limit will not be considered here.

In summary, in the natural scenario of commensurate mass spectrum in the heavy sector, $B_{\mu \rightarrow e}^{Z}$ and $B_{\mu \rightarrow e \gamma}$ are driven by a single combination of heavy neutrino masses and mixing parameters, which we defined as $g_{\text {lfv }}$. Moreover, $B_{\mu \rightarrow e}^{Z}$ and $B_{\mu \rightarrow e \gamma}$ depend only on four independent parameters $\left(g_{\mathrm{lfv}}, M_{W_{2}}, M_{\delta_{L}^{++}}, M_{\delta_{R}^{++}}\right)$, and have the generic structure

$$
\begin{aligned}
& B_{i}=\left|g_{\text {lfv }}\right|^{2} \frac{M_{W_{1}}^{4}}{M_{W_{2}}^{4}} \times \\
& f_{i}\left(\log \frac{M_{W_{2}}}{\sqrt{-q^{2}}}, \quad \log \frac{M_{W_{2}}}{M_{W_{1}}}, \quad r_{L} \equiv \frac{M_{\delta_{L}^{++}}}{M_{W_{2}}}, \quad r_{R} \equiv \frac{M_{\delta_{R}^{++}}}{M_{W_{2}}}\right) .
\end{aligned}
$$

We shall next explore the consequences of such simplified form.

\section{B. $\mu \rightarrow e$ Conversion Versus $\mu \rightarrow e \gamma$}

The first important consequence is that the ratio $R^{A} \equiv$ $B_{\mu \rightarrow e}^{A} / B_{\mu \rightarrow e \gamma}$ does not depend on $g_{\text {lfv }}$, and is a function of $\log \left(M_{W_{2}} / M_{W_{1}}\right), r_{L}, r_{R}$. Our explicit analysis shows that, for input parameters in the commensurate range $0.2 \leq$

\footnotetext{
${ }^{5}$ In the case of $A_{L}$, the relevant conditions are $\left|S_{3}(x)\right| \ll x / 3$, and $\left|S_{3}^{\prime}(x)\right| \ll 1 / 3$.
} 
$r_{L, R} \leq 5, R^{A}$ varies at most by $30 \%$ for any fixed value of $M_{W_{2}} / M_{W_{1}}$. As illustration, in Fig. 4 we show the ratio $R$ for aluminum $R^{A l}$ as a function of $M_{W_{2}} / M_{W_{1}}$ for a range of $r_{L}$ values (the variation with $r_{R}$ is much smaller). The most striking feature of our result is the near independence on the heavy mass parameters (as long as they stay in the natural range), leading to a distinctive prediction of the LRSM for $R^{A l}$. This ratio is of $\mathcal{O}(1)$ in this model and it is naturally confined between 1 and 2 , as shown by the gray area in Fig. 4. The absolute scale on this plot can be understood as a consequence of the logarithmic enhancement of the anapole form factor contributing to $B_{\mu \rightarrow e}$. Different values of $R^{A}$ (in particular values smaller than unity) can be hardly accommodated without unnatural tuning of mass parameters. Indeed, for mass parameters just above the present direct limits $\left(M_{W_{2}}=0.8 \mathrm{TeV}\right.$ and $M_{\delta_{L, R}^{++}}=200 \mathrm{GeV}$ ), we find $R^{A l}=0.8$, which can be considered the minimal acceptable value within this model. This prediction is substantially different from R-parity conserving SUSY scenarios, and can be hopefully tested by future measurements of $B_{\mu \rightarrow e}^{A l}(\mathrm{MECO})$ and $B_{\mu \rightarrow e \gamma}$ (MEG).

The qualitative features encountered in the analysis of $R^{A l}$ apply to other elements as well. In particular, the ratio $R^{A}$ is always of $\mathcal{O}(1)$. We have studied a few more examples, in the same range of mass parameters used above, finding:

$$
R^{T i}: 2 \rightarrow 3.5, \quad R^{A u}: 2 \rightarrow 4, \quad R^{P b}: 1.5 \rightarrow 3 .
$$

\section{C. $\boldsymbol{\mu} \rightarrow \boldsymbol{e}$ Conversion Versus $\boldsymbol{\mu} \rightarrow \mathbf{3 e}$}

Under slightly stronger assumptions, it is also possible to derive an order-of-magnitude relation between $B_{\mu \rightarrow 3 e}$ and $B_{\mu \rightarrow e}$. Assuming dominance of logarithmic terms induced by doubly-charged Higgs diagrams,

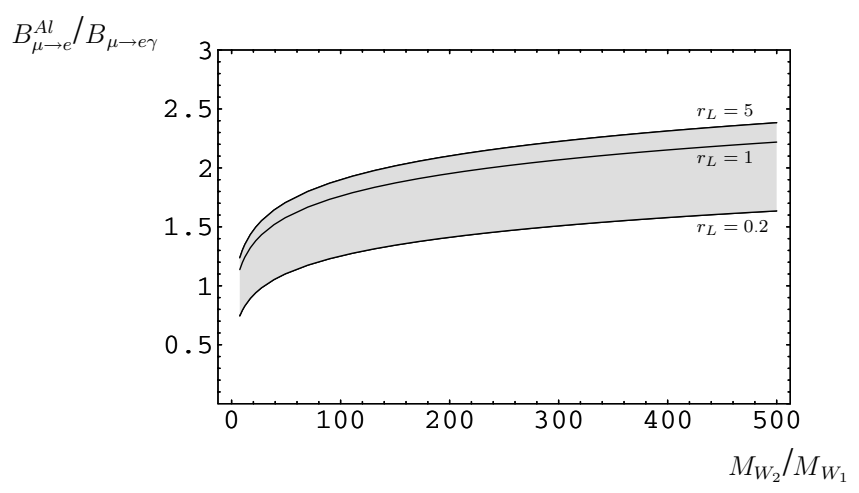

FIG. 4. $\quad R^{A l} \equiv B_{\mu \rightarrow e}^{A l} / B_{\mu \rightarrow e \gamma}$ as a function of $M_{W_{2}} / M_{W_{1}}$, for different values of $r_{L}$. We keep $r_{R}=1$, because the variation of $R^{A l}$ with this parameter is considerably smaller then the variation with $r_{L}$. The shaded band can be considered a prediction of left-right symmetry for $R^{A l}$, assuming commensurate heavy sector. and using $\log \left(M_{\delta_{R}^{++}}^{2} / m_{\mu}^{2}\right) \approx \log \left(M_{\delta_{L}^{++}}^{2} / m_{\mu}^{2}\right)$, one can write

$$
\begin{aligned}
B_{\mu \rightarrow e}= & \frac{8 G_{F}^{2} \alpha^{2}}{9 \pi^{2}} \frac{\left(V^{(p)}\right)^{2}}{\Gamma_{\mathrm{capt}}}\left(\frac{M_{W_{1}}^{4}}{M_{\delta_{L}^{++}}^{4}}+\frac{M_{W_{1}}^{4}}{M_{\delta_{R}^{++}}^{4}}\right) \\
& \times\left(\log \frac{M_{\delta_{R}^{++}}^{2}}{m_{\mu}^{2}}\right)^{2}\left|h_{\mu e} h_{e e}^{*}+h_{\mu \mu} h_{\mu e}^{*}+h_{\mu \tau} h_{\tau e}^{*}\right|^{2} .
\end{aligned}
$$

Under the assumption that $h_{\mu e} h_{e e}^{*} \sim h_{\mu \mu} h_{\mu e}^{*} \sim h_{\mu \tau} h_{\tau e}^{*}$, and that no cancellations occur between the three contributions, one then expects

$$
B_{\mu \rightarrow e}=k_{f} \frac{16 G_{F}^{2} \alpha^{2}}{9 \pi^{2}} \frac{\left(V^{(p)}\right)^{2}}{\Gamma_{\text {capt }}}\left(\log \frac{M_{\delta_{R}^{++}}^{2}}{m_{\mu}^{2}}\right)^{2} B_{\mu \rightarrow 3 e},
$$

where $k_{f}=\left|g_{\text {lfv }}\right|^{2} /\left|h_{\mu e} h_{e e}^{*}\right|^{2}$ is a number of order 1 . For $M_{\delta_{R}^{++}}^{2} \approx M_{\delta_{L}^{++}}^{2} \approx 1 \mathrm{TeV}$, this translates into

$$
B_{\mu \rightarrow 3 e} \sim \frac{3 \times 10^{2}}{k_{f}} B_{\mu \rightarrow e}^{A l} .
$$

So, within this model, one expects that $\mu \rightarrow 3 e$ could be the first rare muon decay to be observed. Sizable deviations from the above pattern would provide information about the parameters $h_{\mu l} h_{l e}^{*}$. In particular, $B_{\mu \rightarrow e}^{A l} / B_{\mu \rightarrow 3 e} \gg 10^{-2}$ would imply dominance of the $l=$ $\mu$ and/or $l=\tau$ contribution in $\left|\sum_{l} h_{\mu l} h_{l e}^{*}\right|$, and may lead to observable signals in $\tau \rightarrow l_{a} l_{b} \bar{l}_{c}$ decays. On the other hand, $B_{\mu \rightarrow e}^{A l} / B_{\mu \rightarrow 3 e} \ll 10^{-3}$ would signal nontrivial relative phases among the couplings, necessary to suppress $\left|\sum_{l} h_{\mu l} h_{l e}^{*}\right|$ compared to $\left|h_{\mu e} h_{e e}^{*}\right|$.

\section{Constraints on heavy neutrino masses and mixing}

LFV in muon decays is driven by $g_{\text {Ifv }}$ and the couplings $h_{i j}$, related to heavy neutrino masses and mixing angles through Eqs. (24) and (25). We now explore the correlations between $g_{\mathrm{lfv}}$ and heavy mass parameters implied by present experimental limits and future limits/observations of $B_{\mu \rightarrow e}^{Z}, B_{\mu \rightarrow e \gamma}$. Subsequently, we discuss the constraints on $h_{\mu e} h_{e e}^{*}$ implied by limits on $B_{\mu \rightarrow 3 e}$.

In order to illustrate the generic model expectations for $\mu \rightarrow e$ conversion and $\mu \rightarrow e \gamma$, we show below approximate expressions for the rates (obtained by setting $r_{L}=$ $\left.r_{R}=1\right)$,

$$
\begin{gathered}
B_{\mu \rightarrow e \gamma}=1.5 \times 10^{-7}\left|g_{\mathrm{lfv}}\right|^{2}\left(\frac{1 \mathrm{TeV}}{M_{W_{2}}}\right)^{4}, \\
B_{\mu \rightarrow e}^{A, Z}=X_{A} \times 10^{-7}\left|g_{\mathrm{lfv}}\right|^{2}\left(\frac{1 \mathrm{TeV}}{M_{\delta_{L, R}^{++}}}\right)^{4} \alpha\left(\log \frac{M_{\delta_{L, R}^{++}}^{2}}{m_{\mu}^{2}}\right)^{2},
\end{gathered}
$$


where $X_{A}$ is the nucleus dependent numerical factor (we find $X_{A}=0.8,1.3,1.6$, and 1.1 for $\mathrm{Al}, \mathrm{Ti}, \mathrm{Au}$, and $\mathrm{Pb}$, respectively). These branching ratios have to be compared with present experimental limits:

$$
\begin{aligned}
B_{\mu \rightarrow e \gamma} & <1.2 \times 10^{-11}[3], B_{\mu \rightarrow e}^{T i}<4.3 \times 10^{-12}[6], B_{\mu \rightarrow e}^{A u} \\
& <8 \times 10^{-13}[4], B_{\mu \rightarrow e}^{P b}<4.6 \times 10^{-11[7]} .
\end{aligned}
$$

Thus, assuming commensurate spectrum and $g_{\text {lfv }} \sim 1$ (i.e., large mixing angles and nondegenerate heavy neutrinos), consistency with present limits implies that the scale of $S U(2)_{R}$ breaking has to be around $20 \mathrm{TeV}$. On the other hand, for $M_{W_{2}}$ in the 1-10 TeV range, present experimental limits already impose nontrivial constraints on $g_{\text {lfv }}$ (left panel in Fig. 5). Values of $g_{\text {lfv }}$ at the $10^{-2}-10^{-3}$ level imply either small mixing an-
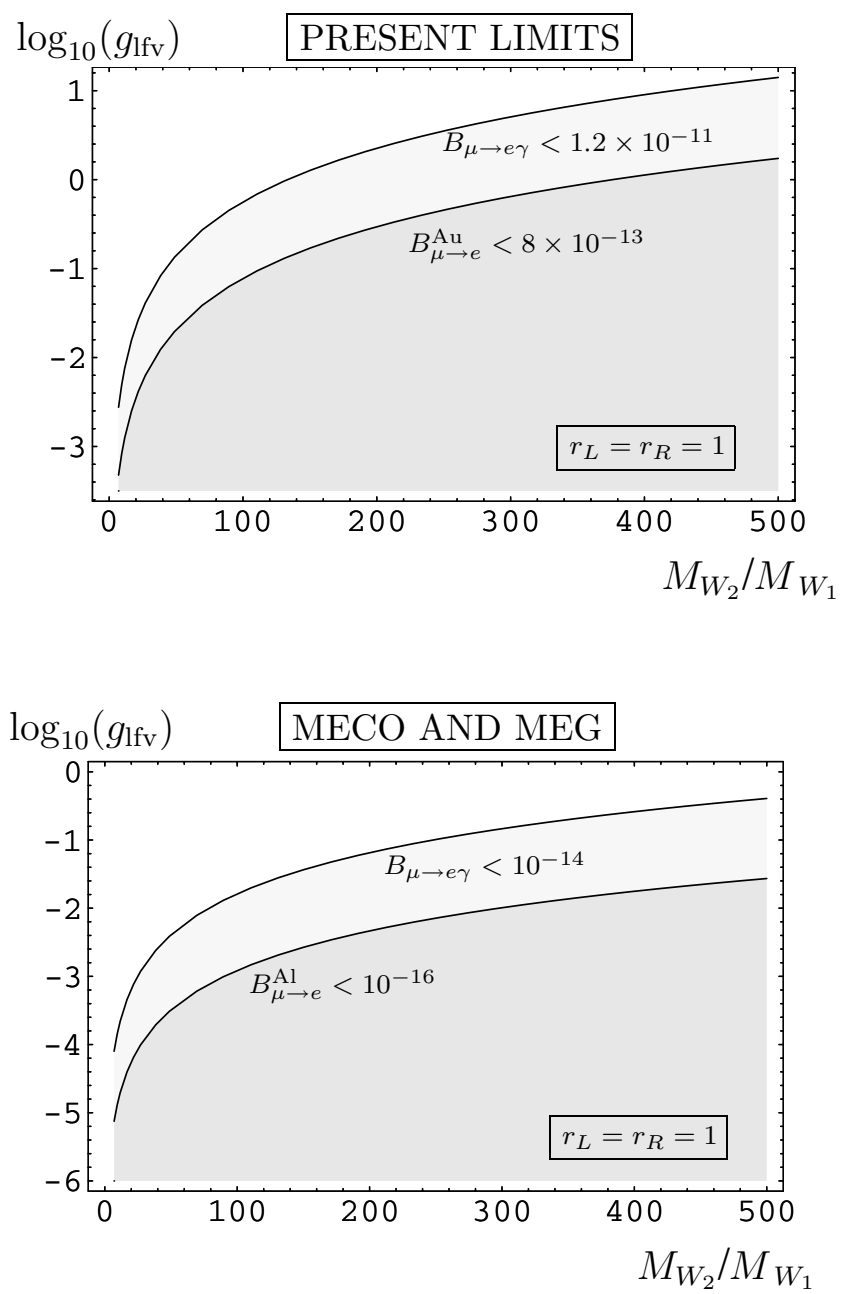

FIG. 5 (color online). Correlations in the $g_{1 \mathrm{fv}}-\left(M_{W_{2}} / M_{W_{1}}\right)$ plane imposed by present and future (MEG and MECO) limits on $B_{\mu \rightarrow e}$ and $B_{\mu \rightarrow e \gamma}$. The shaded area represents the region allowed by limits reported on the plot. In this plot we use $r_{L}=$ $r_{R}=1$. Lowering $r_{L}$ and/or $r_{R}$ poses tighter constraints on $g_{\text {lfv }}$, for fixed $M_{W_{2}}$. gles in the heavy neutrino sector or nearly degenerate heavy neutrinos, on the scale set by $M_{W_{2}}$. The most stringent constraints at present come from $\mu \rightarrow e$ conversion in gold. Future experiments MEG [8] and MECO [9] will be able to probe even higher mass scales and put more stringent upper limits on $g_{\text {lfv }}$ (right panel in Fig. 5). Once again, $\mu \rightarrow e$ conversion will probe the model parameter space more strongly.

Focusing on $\mu \rightarrow e$ conversion (present limits and projected MECO sensitivity), in Fig. 6 we report a more detailed study of the constraints. At fixed $M_{W_{2}}$, lowering or raising $r_{L, R}$ within the natural range $0.2 \lesssim$ $r_{L, R} \lesssim 5$, can change the bound on $g_{\text {lfv }}$ by an order-of magnitude. Lighter Higgs particles imply tighter upper
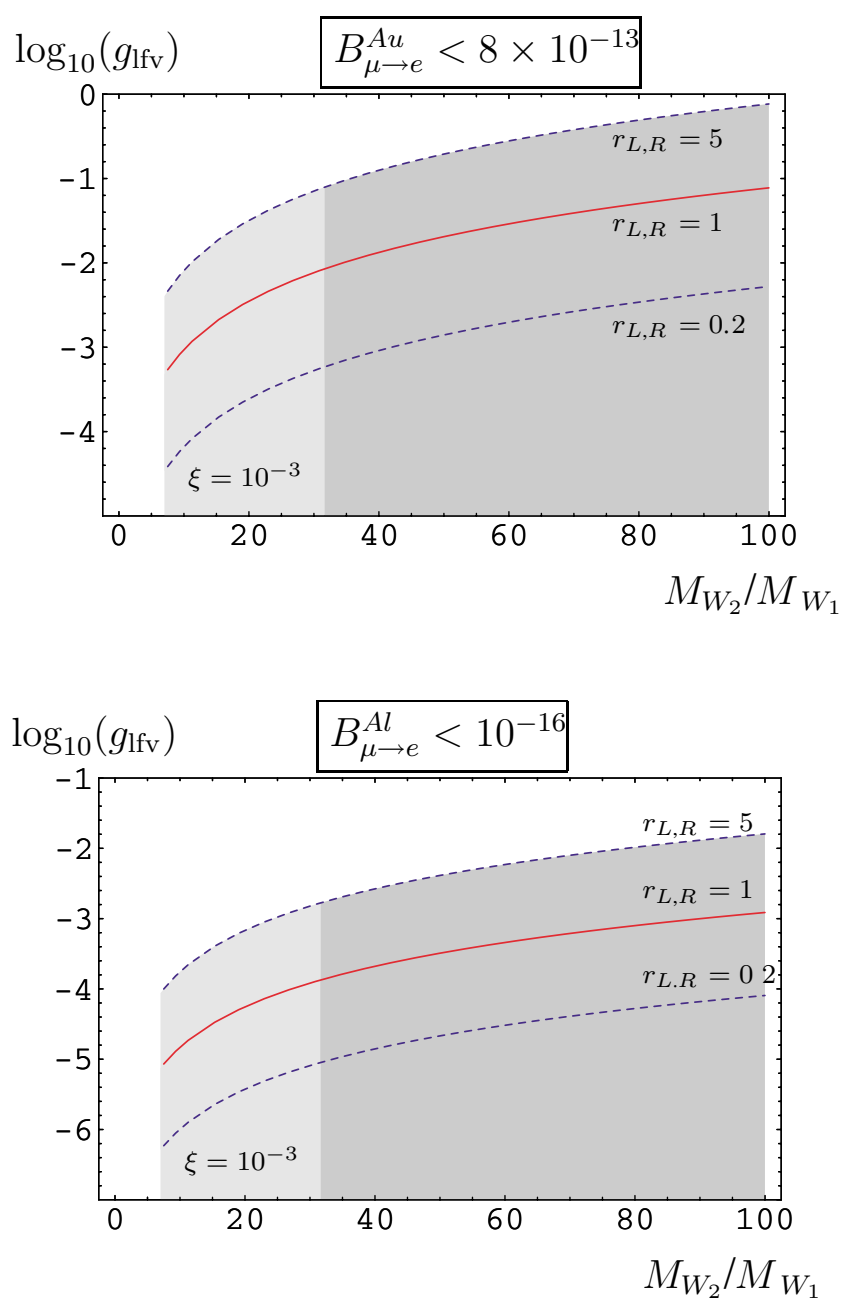

FIG. 6 (color online). Correlations in the $g_{\text {lfv }}-\left(M_{W_{2}} / M_{W_{1}}\right)$ plane imposed by $B_{\mu \rightarrow e}$, before and after MECO's goal has been reached, for different values of $r_{L}=r_{R}$. The shaded area represents the region allowed by the assumed limits on $B_{\mu \rightarrow e}$. A nonzero mixing angle $\xi$ would further reduce the allowed region. As an illustration, the allowed region for $\xi=10^{-3}$ is plotted in light-gray. 
limits on $g_{\text {lfv }}$. Finally, the impact of a nonzero mixing angle $\xi$ (detectable, for example, through right-handed current signals in $\beta$ decays) is also considered in Fig. 6. A nonvanishing $\xi$ would imply [37] the upper bound
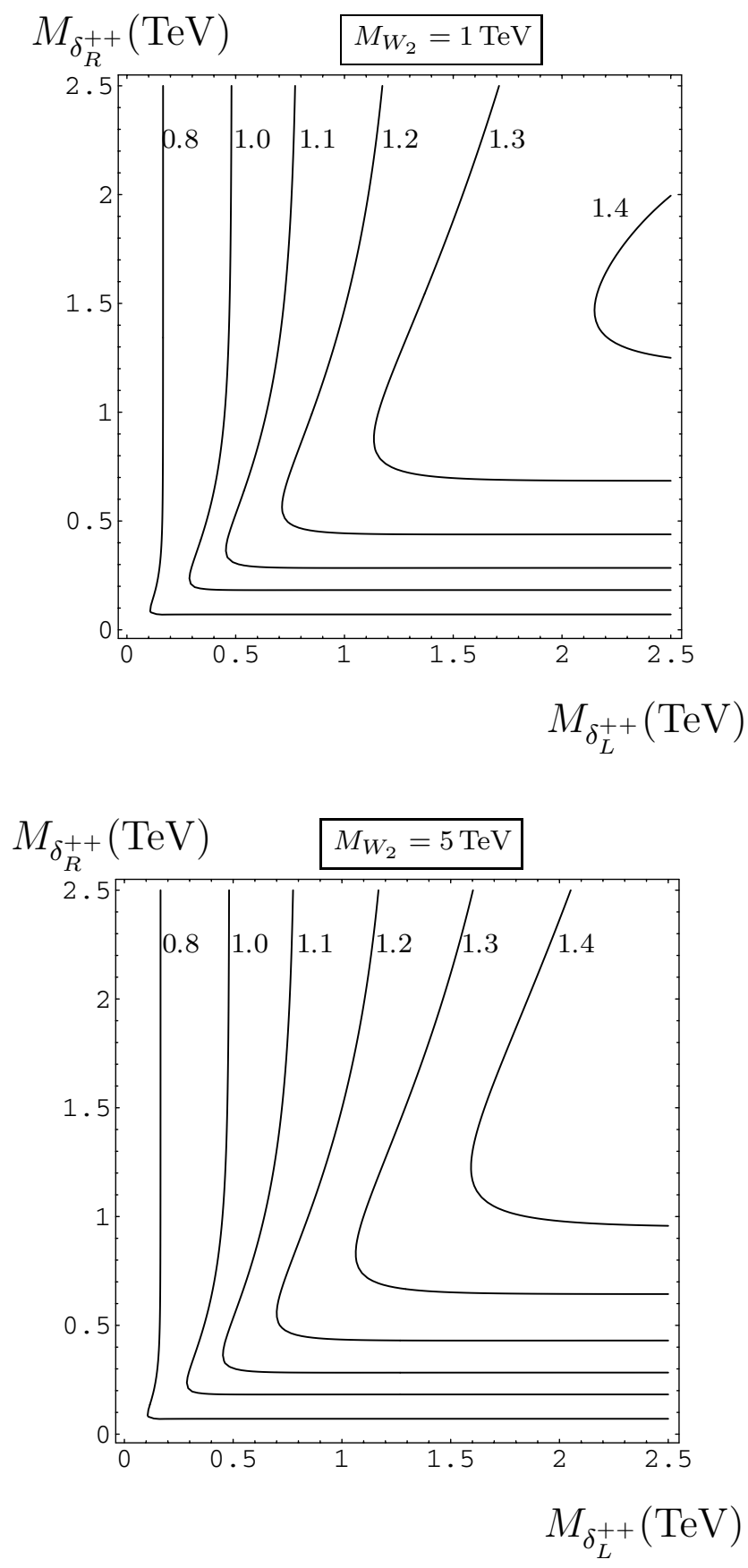

FIG. 7. Contour plot of $R^{A l} \equiv B_{\mu \rightarrow e}^{\mathrm{Al}} / B_{\mu \rightarrow e \gamma}$ in the $M_{\delta_{L}^{++}}$ $-M_{\delta_{R}^{++}}$plane, for $M_{W_{2}}=1 \mathrm{TeV}$ (top panel) and for $M_{W_{2}}=$ $5 \mathrm{TeV}$ (bottom panel). Each curve is labeled by the corresponding $R^{A l}$. As a function of the Higgs mass along the line $M_{\delta_{L}^{++}}=$ $M_{\delta_{R}^{++}}, R^{A l}$ reaches a maximum at $M_{\delta_{L}^{++}} \sim 2 M_{W_{2}}$ and then decreases, due to decoupling of doubly-charged Higgs bosons (the latter effect is not visible in the plots).
$M_{W_{2}} / M_{W_{1}} \leq 1 / \sqrt{\xi}$, and thus narrow down the allowed region in the $g_{\text {lfv }}-M_{W_{2}} / M_{W_{1}}$ plane (light-gray region in Fig. 6).

Additional information on heavy neutrino parameters can be obtained in principle from $\mu \rightarrow 3 e$. The rate depends on doubly-charged Higgs masses and the combination $\left|h_{\mu e} h_{e e}^{*}\right|$ (Eq. (53)). The present limit $B_{\mu \rightarrow 3 e}<$ $10^{-12}$ [31] implies (assuming $M_{\delta_{L}^{++}}=M_{\delta_{R}^{++}}{ }^{6}$

$$
\left|h_{\mu e} h_{e e}^{*}\right| \leq 1.55 \times 10^{-4} \sqrt{\frac{B_{\mu \rightarrow 3 e}}{10^{-12}}}\left(\frac{M_{\delta_{L, R}^{++}}}{1 \mathrm{TeV}}\right)^{2} .
$$

Thus, assuming $M_{\delta^{++}} \sim 1 \mathrm{TeV}$, the couplings $h_{i j}$ are constrained to be at the $\sim 10^{-2}$ level. Unlike the case of $g_{\text {lfv }}$, however, the smallness of $h_{\mu e}$ does not imply small mixing angles or almost-degenerate heavy neutrinos, be cause the Majorana phases contained in $K_{R}$ may lead to cancellations in the sum of Eq. (24).

\section{E. Testing the model: interplay with collider mea- surements}

As noted above, information from LFV processes and other aspects of low-energy phenomenology (such as signals of right-handed currents) can severely constrain the model parameter space in the near future. Moreover, given that $B_{\mu \rightarrow e}$ and $B_{\mu \rightarrow e \gamma}$ depend only on $g_{\text {lfv }}, M_{W_{2}}, M_{\delta_{L}^{++}}, M_{\delta_{R}^{++}}$, collider searches of heavy particles and low-energy searches of LFV decays jointly provide a powerful probe of left-right symmetry. In fact, in the best-case scenario, separate measurements of $B_{\mu \rightarrow e}^{A l}$, $B_{\mu \rightarrow e \gamma}$ and the mass parameters $M_{W_{2}}, M_{\delta_{L}^{++}}, M_{\delta_{R}^{++}}$would allow one to test the model (4 parameters versus $5 \mathrm{ob}$ servables). Even in less optimistic scenarios, one can imagine using collider information to narrow down the model predictions for LFV processes, or use observation of LFV to determine allowed regions in the heavy mass parameter space.

As a simple illustration of this point, we show in Fig. 7 contour plots of $R^{A l} \equiv B_{\mu \rightarrow e}^{A l} / B_{\mu \rightarrow e \gamma}$ in the $M_{\delta_{L}^{++}} M_{\delta_{R}^{++}}$ plane, for two values of $M_{W_{2}}$. We focus on the case of heavy masses in the 1-2 TeV range, which will be accessible at the LHC and Tevatron II [41]. In this mass-region, the model expectations are almost independent of $M_{W_{2}}$. Moreover, one sees that values of $R^{A l}<0.8$ can only occur for $M_{\delta_{L, R}^{++}}<100 \mathrm{GeV}$, already excluded by direct searches. Depending on future experimental developments, possible uses of the plots in Figs. 7 include:

\footnotetext{
${ }^{6} \mathrm{~A}$ weaker upper limit on the same combination of parameters can be derived from searches of muonium antimuonium transition [38,39]. In general, present limits on the flavor diagonal coupling $h_{e e}$ from Bhabha scattering [40], and other combinations of $h_{i j}$ from rare $\tau$ decays are much weaker (typically $B_{\tau \rightarrow l_{a} l_{b} l_{c}}<10^{-6}[31]$ ).
} 
(i) Given measurements of Higgs and heavy gauge boson masses, one can infer rather precisely where to expect $R^{A l}$ within this scenario.

(ii) Given an experimental signal for $B_{\mu \rightarrow e}^{A l}$ and $B_{\mu \rightarrow e \gamma}$, one can identify the allowed region in the $M_{\delta^{++}}-M_{\delta_{R}^{++}}$plane, for different values of $M_{W_{2}}$. Collider searches could then confirm or falsify the model expectations. As can be seen from the plots, however, in order to have a significant test, the fractional uncertainty on $R^{A l}$ should be at most $20 \%$ (otherwise most of the $M_{\delta_{L}^{++}} M_{\delta_{R}^{++}}$would be allowed). Given the projected sensitivities, this may be achieved at the next generation experiments if $B_{\mu \rightarrow e \gamma} \geq 2$. $\times 10^{-13}$.

\section{CONCLUSIONS}

The study of flavor violation among leptons now lies at the forefront of particle and nuclear physics. The tiny masses of the three lightest neutrinos and the nearly maximal mixing among them stands in stark contrast with the situation involving quarks, and the origin of this difference remains a fundamental and unsolved puzzle. A variety of scenarios have been proposed that attempt to answer this question, and these ideas would have predictable consequences for other observables. In this study, we have analyzed the consequences of one such scenario-the left-right symmetric model-that entails a minimal extension of the SM gauge symmetries and that includes nonsterile, right-handed neutrinos whose mass could be generated at the multi-TeV scale, albeit with some fine-tuning. We have shown how it implies relationships among various LFV decays of the muon that could distinguish it experimentally from other models of LFV. We have also illustrated how direct searches for right-handed gauge bosons and triplet Higgs at the Tevatron and LHC would complement the charged lepton LFV studies and either help favor or rule out the possibility of rather low-scale LFV without SUSY.

The main conclusions of our study are:

(i) The branching ratios $B_{\mu \rightarrow e}$ and $B_{\mu \rightarrow e \gamma}$ are similar in magnitude, in distinction to other possible scenarios which predict that $B_{\mu \rightarrow e} / B_{\mu \rightarrow e \gamma} \sim \alpha$.

(ii) Within the LRSM, and with reasonable additional assumptions, $B_{\mu \rightarrow 3 e} / B_{\mu \rightarrow e} \sim 300$, making the process $\mu \rightarrow 3 e$ perhaps easiest to observe.

(iii) The existing limits on the LFV muon decays already substantially constrain the mixing and mass splittings of the heavy right-handed neutrinos. The planned more sensitive experiments will therefore test the LRSM severely.

If the LRSM scenario turns out to be correct, the deeper connections between the heavy and light neutrino spectrum would, then, have to be pursued by additional experimental and theoretical work. On the other hand, should experiment eliminate the possibility of nonsupersymmetric, low-scale LFV based on the considerations discussed above, the lepton flavor problem will nevertheless remain a rich area of study, both theoretically and experimentally, for some time to come.

\section{ACKNOWLEDGMENTS}

We thank M. B Wise for useful comments provided during the course of carrying out this calculation. This work was supported in part under U.S. Department of Energy contract No. DE-FG03-88ER40397 and NSF Grant No. PHY-0071856.

\section{APPENDIX A: LOOP FUNCTIONS}

We collect here the functions $S_{i}(x)$ and $D_{1}(x, y)$ appearing in the expression of various $\mu \rightarrow e$ form factors.

$$
\begin{aligned}
S_{1}(x)= & \frac{4 x}{(1-x)^{2}}\left[6-7 x+x^{2}+(2+3 x) \log x\right], \\
S_{2}(x)= & \frac{x(4-3 x)}{(1-x)^{2}}-\frac{2 x\left(12-10 x+x^{2}\right)}{3(1-x)^{2}}\left[S_{4}(x)+1\right], \\
S_{3}(x)= & -\frac{x(1+2 x)}{8(1-x)^{2}}+\frac{3 x^{2}}{4(1-x)^{2}}\left[S_{4}(x)+1\right], \\
D_{1}(x, y)= & x\left(2-\log \frac{y}{x}\right) \\
& +\frac{\left(-8 x+9 x^{2}-x^{3}\right)+\left(-8 x^{2}+x^{3}\right) \log x}{(1-x)^{2}}(1-x+\log x), \\
& +\frac{x\left(y-y^{2}+y^{2} \log y\right)}{(1-y)^{2}}+\frac{2 x y(4-x) \log x}{(1-x)(1-y)} \\
& +\frac{2 x(x-4 y) \log (y / x)}{(x-y)(1-y)} .
\end{aligned}
$$

Both $S_{i}(x)$ and $D_{1}(x, y)$ are regular at $x=1$ and $y=1$. Note that the potentially dangerous contribution involving the large mass-ratio $y_{n}=\left(M_{n} / M_{W_{1}}\right)^{2}$ has a finite limit for $y_{n} \rightarrow \infty$ :

$$
\lim _{y \rightarrow \infty} D_{1}(x, y)=-7 S_{4}(x) .
$$

\section{APPENDIX B: FULL EXPRESSIONS FOR $\mu e \gamma^{*}$ FORM FACTORS}

In terms of the interactions vertices reported in Eq. (21) and (22), and without neglecting the charged 
lepton mass-dependence of loops, the photonic form factors read:

$$
\begin{aligned}
F_{R}^{(\gamma)}= & \sum_{n=\text { heavy }}\left(K_{R}^{\dagger}\right)_{e n}\left(K_{R}\right)_{n \mu} \frac{M_{W_{1}}^{2}}{M_{W_{2}}^{2}} S_{2}\left(x_{n}\right) \\
& +\sum_{l=e, \mu, \tau} h_{e l}^{*} h_{l \mu} \frac{M_{W_{1}}^{2}}{M_{\delta_{R}^{++}}^{2}} \\
& \times\left[-\frac{40}{9}-\frac{8}{3} \log \left(\frac{-q^{2}}{M_{\delta_{R}^{++}}^{2}}\right)-16 S_{5}\left(\frac{m_{l}^{2}}{-q^{2}}\right)\right], \\
F_{L}^{(\gamma)}= & \sum_{l=e, \mu, \tau} h_{e l}^{*} h_{l \mu} \frac{M_{W_{1}}^{2}}{M_{\delta_{L}^{++}}^{2}} \\
& \times\left[-\frac{40}{9}-\frac{8}{3} \log \left(\frac{-q^{2}}{M_{\delta_{L}^{++}}^{2}}\right)-16 S_{5}\left(\frac{m_{l}^{2}}{-q^{2}}\right)\right] \\
& -\frac{2}{9}\left(\tilde{h}^{\dagger} \tilde{h}\right)_{e \mu} \frac{M_{W_{1}}^{2}}{M_{\delta_{L}^{+}}^{2}},
\end{aligned}
$$

$$
\begin{aligned}
& 16 \pi^{2} A_{L}= \sum_{n=\text { heavy }}\left(K_{R}^{\dagger}\right)_{e n}\left(K_{R}\right)_{n \mu} \frac{M_{W_{1}}^{2}}{M_{W_{2}}^{2}} S_{3}\left(x_{n}\right) \\
&-\frac{1}{3} \sum_{l=e, \mu, \tau} h_{e l}^{*} h_{l \mu} \frac{M_{W_{1}}^{2}}{M_{\delta_{R}^{++}}^{2}}, \\
& 16 \pi^{2} A_{R}=-\frac{1}{3} \sum_{l=e, \mu, \tau} h_{e l}^{*} h_{l \mu} \frac{M_{W_{1}}^{2}}{M_{\delta_{L}^{++}}^{2}}-\frac{1}{24}\left(\tilde{h}^{\dagger} \tilde{h}\right)_{e \mu} \frac{M_{W_{1}}^{2}}{M_{\delta_{L}^{+}}^{2}}
\end{aligned}
$$

where the function $S_{5}(x)$ is:

$$
S_{5}(x)=\int_{0}^{1} d y y(1-y) \log [x+y(1-y)] .
$$

[1] S. Lokanthan and J. Steinberger, Phys. Rev. A 98, 240 (1955).

[2] G. Feinberg, Phys. Rev. 110, 1482 (1958).

[3] M. L. Brooks et al. Phys. Rev. Lett. 83, 1521 (1999).

[4] W. Bertl et al. PSI (2002) (unpublished)9.

[5] U. Bellgardt et al. Nucl. Phys. B 299, 1 (1988).

[6] C. Dohmen et al. Phys. Lett. B 317, 631 (1993).

[7] W. Honecker et al. Phys. Rev. Lett. 76, 200 (1996).

[8] G. Signorelli, J. Phys. G 29, 2027 (2003).

[9] J. L. Popp, Nucl. Instrum. Methods Phys. Res., Sect. A 472, 354 (2000).

[10] F. Borzumati and A. Masiero, Phys. Rev. Lett. 57, 961 (1986).

[11] G. K. Leontaris, K. Tamvakis, and J. D. Vergados, Phys. Lett. B 171, 412 (1986).

[12] J. Hisano, T. Moroi, K. Tobe, M. Yamaguchi, and T. Yanagida, Phys. Lett. B 357, 579 (1995).

[13] R. Barbieri and L. J. Hall, Phys. Lett. B 338, 212 (1994).

[14] R. Barbieri, L. J. Hall, and A. Strumia, Nucl. Phys. B 445, 219 (1995).

[15] K. Huitu, J. Maalampi, M. Raidal, and A. Santamaria, Phys. Lett. B 430, 355 (1998).

[16] A. de Gouvea, S. Lola, and K. Tobe, Phys. Rev. D 63, 035004 (2001).

[17] Riazuddin, R. E. Marshak, and R. N. Mohapatra, Phys. Rev. D 24, 1310 (1981).

[18] G. Barenboim and M. Raidal, Nucl. Phys. B 484, 63 (1997).

[19] N. G. Deshpande, J. F. Gunion, B. Kayser, and F. I. Olness, Phys. Rev. D 44, 837 (1991).

[20] R. N. Mohapatra, hep-ph/0306016.
[21] J. C. Pati and A. Salam, Phys. Rev. D 10, 275 (1974); R. N. Mohapatra and J. C. Pati, Phys. Rev. D 11, 566 (1975); G. Senjanovic and R. N. Mohapatra, Phys. Rev. D 12, 1502 (1975).

[22] R. N. Mohapatra and G. Senjanovic, Phys. Rev. Lett. 44, 912 (1980).

[23] R. N. Mohapatra and G. Senjanovic, Phys. Rev. D 23, 165 (1981).

[24] G. B. Gelmini and M. Roncadelli, Phys. Lett. B 99, 411 (1981).

[25] M. Raidal and A. Santamaria, Phys. Lett. B 421, 250 (1998).

[26] T. Appelquist and J. Carazzone, Phys. Rev. D 11, 2856 (1975).

[27] Y. Grossman and S. Rakshit, Phys. Rev. D 69, 093002 (2004).

[28] P. Duka, J. Gluza, and M. Zralek, Ann. Phys. (N.Y.) 280, 336 (2000).

[29] G. Barenboim, M. Gorbahn, U. Nierste, and M. Raidal, Phys. Rev. D 65, 095003 (2002).

[30] D. N. Spergel et al., Astrophys. J. Suppl. Ser. 148, 175 (2003).

[31] Particle Data Group Collaboration, K. Hagiwara et al., Phys. Rev. D 66, 010001 (2002).

[32] Ya. B. Zeldovich, Zh. Eksp. Teor. Fiz. 33, 1531 (1957); [Sov. Phys. JETP 6, 1184 (1958)]; Zh. Eksp. Teor. Fiz.39, 115 (1960); [Sov. Phys. JETP 12, 177 ( 1961)]

[33] R. Kitano, M. Koike, and Y. Okada, Phys. Rev. D 66, 096002 (2002) .

[34] A. Czarnecki, W. J. Marciano, and K. Melnikov, hep-ph/ 9801218. 
[35] G. Prezeau, M. Ramsey-Musolf, and P. Vogel, Phys. Rev. D 68, 034016 (2003).

[36] R. N. Mohapatra, Phys. Rev. D 34, 909 (1986).

[37] E. Masso, Phys. Rev. Lett. 52, 1956 (1984).

[38] R. Abela et al., Phys. Rev. Lett. 77, 1950 (1996).

[39] P. Herczeg and R. N. Mohapatra, Phys. Rev. Lett. 69, 2475
(1992).

[40] M. Kuze and Y. Sirois, Prog. Part. Nucl. Phys. 50, 1 (2003).

[41] A. Datta and A. Raychaudhuri, Phys. Rev. D 62, 055002 (2000). 